\title{
More Reliable Protein NMR Peak Assignment via Improved 2-Interval Scheduling
}

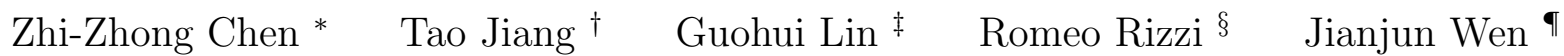 \\ Dong $\mathrm{Xu} \| \quad$ Ying $\mathrm{Xu}{ }^{* *}$
}

\begin{abstract}
Protein NMR peak assignment refers to the process of assigning a group of "spin systems" obtained experimentally to a protein sequence of amino acids. The automation of this process is still an unsolved and challenging problem in NMR protein structure determination. Recently, protein backbone NMR peak assignment has been formulated as an interval scheduling problem, where a protein sequence $\mathcal{P}$ of amino acids is viewed as a discrete time interval $\mathcal{I}$ (the amino acids on $\mathcal{P}$ one-to-one correspond to the time units of $\mathcal{I}$ ), each subset $S$ of spin systems that are known to originate from consecutive amino acids of $\mathcal{P}$ is viewed as a "job" $j_{S}$, the preference of assigning $S$ to a subsequence $P$ of consecutive amino acids on $\mathcal{P}$ is viewed as the profit of executing job $j_{S}$ in the subinterval of $\mathcal{I}$ corresponding to $P$, and the goal is to maximize the total profit of executing the jobs (on a single machine) during $\mathcal{I}$. The interval scheduling problem is Max SNP-hard in general. Typically the jobs that require one or two consecutive time units are the most difficult to assign/schedule. To solve these most difficult assignments, we present an efficient $\frac{13}{7}$-approximation algorithm. Combining this algorithm with a greedy filtering strategy for handling long jobs (i.e. jobs that need more than two consecutive time units), we obtained a new efficient heuristic for protein NMR peak assignment. Our study using experimental data shows that the new heuristic produces the best peak assignment in most of the cases, compared with the NMR peak assignment algorithms in the literature. The $\frac{13}{7}$-approximation algorithm is also the first approximation algorithm for a nontrivial case of the classical (weighted) interval scheduling problem that breaks the ratio 2 barrier.
\end{abstract}

Keywords: structural genomics, computational biology, protein NMR peak assignment, approximation algorithm, interval scheduling, constrained bipartite matching.

\section{Introduction}

The NMR (nuclear magnetic resonance) technique is a major method to determine protein structures. A time-consuming bottleneck of this technique is the NMR peak assignment, which usually

* Department of Mathematical Sciences, Tokyo Denki University, Hatoyama, Saitama 350-0394, Japan. Part of work done while visiting at University of Alberta. Email: chen@r.dendai.ac.jp.

${ }^{\dagger}$ Supported in part by NSF Grants CCR-9988353 and ITR-0085910, and National Key Project for Basic Research (973). Department of Compute Science, University of California, Riverside, CA 92521, and Shanghai Center for Bioinformation Technology. Email: jiang@cs.ucr.edu.

${ }^{\ddagger}$ Supported in part by NSERC grants RGPIN249633 and A008599, PENCE, and Startup Grant REE-P5-01-02-Sci from the University of Alberta. Department of Computing Science, University of Alberta, Edmonton, Alberta T6G 2E8, Canada. Email: ghlin@cs.ualberta.ca.

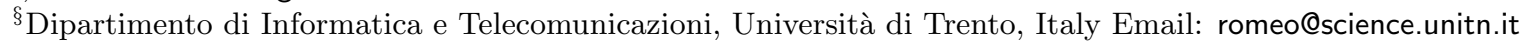

`Department of Computer Science, University of California, Riverside, CA 92521. Email: wjianju@cs.ucr.edu.

"Supported by the Office of Biological and Environmental Research, U.S. Department of Energy, under Contract DE-AC05-00OR22725, managed by UT-Battelle, LLC. Protein Informatics Group, Life Sciences Division, Oak Ridge National Laboratory, Oak Ridge, TN 37831-6480, USA. Email: xud@ornl.gov.

** Supported by the Office of Biological and Environmental Research, U.S. Department of Energy, under Contract DE-AC05-00OR22725, managed by UT-Battelle, LLC. Protein Informatics Group, Life Sciences Division and Computer Sciences and Mathematics Division, Oak Ridge National Laboratory. Oak Ridge, TN 37831-6480, USA. Email: xyn@ornl.gov. 
takes weeks or sometimes even months of manual work to produce a nearly complete assignment. A protein NMR peak assignment is to establish a one-to-one mapping between two sets of data: (1) a group of "spin systems" obtained experimentally, each corresponding to a number of spectra related to the same amino acid; (2) a known protein sequence of amino acids. The automation of the assignment process is still an unsolved and challenging problem in NMR protein structure determination.

Two key pieces of information form the foundation of NMR peak assignment and the starting point of our work:

- The likelihood (or weight) of the matching between a spin system and an amino acid on the protein sequence. The weight can be derived from the statistical distribution of spin systems in different amino acid types and predicted secondary structures [9].

- The sequential adjacency (i.e., consecutivity) information of some subsets of spin systems (i.e., each such subset of spin systems should correspond to a subsequence of consecutive amino acids on the protein sequence). Each maximal such subset is called a segment of spin systems. It is worth noting that each segment usually consists of at most 10 spin systems. The adjacency information can be obtained from experiments.

In a recently developed computational framework [9], the NMR peak assignment problem has been formulated as a (weighted) interval scheduling problem ${ }^{1}$ as follows. A protein sequence $\mathcal{P}$ of amino acids is viewed as a discrete time interval $\mathcal{I}$ (the amino acids on $\mathcal{P}$ one-to-one correspond to the time units of $\mathcal{I}$ ). Each segment $S$ of spin systems is viewed as a job $j_{S}$. Each job $j_{S}$ requires $|S|$ consecutive time units of $\mathcal{I}$ (this corresponds to the requirement that the spin systems in $S$ should be assigned to $|S|$ consecutive amino acids on $\mathcal{P}$ ). For each time unit $t$ of $\mathcal{I}$, the profit $w\left(j_{S}, t\right)$ of starting job $j_{S}$ at time unit $t$ and finishing at time unit $t+|S|-1$ of $\mathcal{I}$ corresponds to the preference (or total weight) of assigning the spin systems in $S$ to those $|S|$ consecutive amino acids on $\mathcal{P}$ that correspond to the time units $t, t+1, \ldots, t+|S|-1$. Given $\mathcal{I}$, the jobs $j_{S}$, and the profits $w\left(j_{S}, t\right)$, our goal is to maximize the total profit of the executed jobs (i.e., we want to find a maximum-likelihood assignment of the given spin systems to the amino acids on $\mathcal{P}$ ).

Unfortunately, the interval scheduling problem is Max SNP-hard [3, 4]. Indeed, for every integer $k \geq 2$, the special case of the interval scheduling problem (called the $k$-interval scheduling problem or $k$-ISP for short), where each job requires at most $k$ consecutive time units, is Max SNP-hard. On the other hand, several 2-approximation algorithms for the interval scheduling problem have been developed $[1,2,3,4]$. Although these algorithms are theoretically sound, applying them to protein NMR peak assignment produces unsatisfactory assignments as demonstrated in [3]. A major reason why these algorithms do not have good performance in protein NMR peak assignment is that they ignore the following important observation:

- In protein NMR peak assignment, long segments of spin systems are typically easier to assign than shorter ones. Indeed, many long segments have obvious matches based on the total matching weights, while assignments of isolated spin systems or segments consisting of only two spin systems are ambiguous.

The above observation suggests the following heuristic framework for protein NMR peak assignment: first try to assign segments consisting of at least $k+1$ spin systems for some small integer $k$ (say, $k=2$ ), and then solve an instance of $k$-ISP. In [7], we have presented such a heuristic and have shown that it is very effective for protein NMR peak assignment. A major drawback of the heuristic in [7] is that it uses an inefficient branch-and-bound algorithm for $k$-ISP.

In order to improve the efficiency of the heuristic in [7], we present a new approximation algorithm for 2-ISP in this paper. This algorithm achieves an approximation ratio of $\frac{13}{7}$ and is the first approximation algorithm for a nontrivial case of the classical interval scheduling problem that

\footnotetext{
${ }^{1}$ In [9] it was called the constrained bipartite matching problem.
} 
breaks the ratio 2 barrier. ${ }^{2}$ Our algorithm is combinatorial and quite nontrivial - it consists of four separate algorithms and outputs the best solution returned by them. The main tool used in the algorithm design is maximum-weight bipartite matching and careful manipulation of the input instance. Substituting the new algorithm for the branch-and-bound algorithm in the heuristic in [7], we obtain a new heuristic for protein NMR peak assignment. ${ }^{3}$ We have performed extensive experiments on 70 instances of NMR data derived from 14 proteins to evaluate the performance of our new heuristic in terms of (i) the weight of the assignment and (ii) the number of correctly assigned resonance peaks. The experimental results show that not only does the new heuristic run very fast, it also produces the best peak assignment on most of the instances, compared with the protein NMR peak assignment algorithms in the recent literature [3, 4, 7, 9].

The rest of the paper is organized as follows. The $\frac{13}{7}$-approximation algorithm for 2-ISP is presented in Section 2. In Section 3, we consider an interesting special profit function in interval scheduling where the profit of executing a job at each specific time interval is either 0 or proportional to the length of the job, ${ }^{4}$ and we present a $(1.5+\epsilon)$-approximation algorithm for 2-ISP under this special profit function for any $\epsilon>0,{ }^{5}$ which improves an approximation result in [4]. In Section 4, we describe our new heuristic for protein NMR peak assignment based on the $\frac{13}{7}$-approximation algorithm for 2-ISP, and give the experimental results. We end this paper with a short discussion in Section 5.

\section{A new approximation algorithm for 2-ISP}

Let $\mathcal{I}$ be the given discrete time interval. Without loss of generality, we may assume that $\mathcal{I}=$ $[0, I]$. Let $\mathcal{J}_{1}=\left\{v_{1}, v_{2}, \ldots, v_{n_{1}}\right\}$ be the given set of jobs requiring one time unit of $\mathcal{I}$. Let $\mathcal{J}_{2}=$ $\left\{v_{n_{1}+1}, v_{n_{1}+3}, \ldots, v_{n_{1}+2 n_{2}-1}\right\}$ be the given set of jobs requiring two contiguous time units of $\mathcal{I}$. Note that $n_{1}+n_{2}$ is the total number of given jobs. For each $1 \leq i \leq I$, let $u_{i}$ denote the time unit $[i-1, i]$ of $\mathcal{I}$. Let $U=\left\{u_{i} \mid 1 \leq i \leq I\right\}$. Let $\mathcal{J}_{2}^{\prime}=\left\{v_{n_{1}+2}, v_{n_{1}+4}, \ldots, v_{n_{1}+2 n_{2}}\right\}$. Let $V=\mathcal{J}_{1} \cup \mathcal{J}_{2} \cup \mathcal{J}_{2}^{\prime}$. We construct an edge-weighted bipartite graph $G$ with color classes $U$ and $V$ as follows: For every $v_{j} \in \mathcal{J}_{1}$ and every $u_{i} \in U$ such that the profit of executing job $v_{j}$ in time unit $u_{i}$ is positive, $\left(u_{i}, v_{j}\right)$ is an edge of $G$ and its weight is the profit. Similarly, for every $v_{j} \in \mathcal{J}_{2}$ and every $u_{i} \in U$ such that the profit of executing job $v_{j}$ in the two time units $u_{i}, u_{i+1}$ is positive, both $\left(u_{i}, v_{j}\right)$ and $\left(u_{i+1}, v_{j+1}\right)$ are edges of $G$ and the weight of each of them is half the profit. Figure 1 shows an example of $G$.

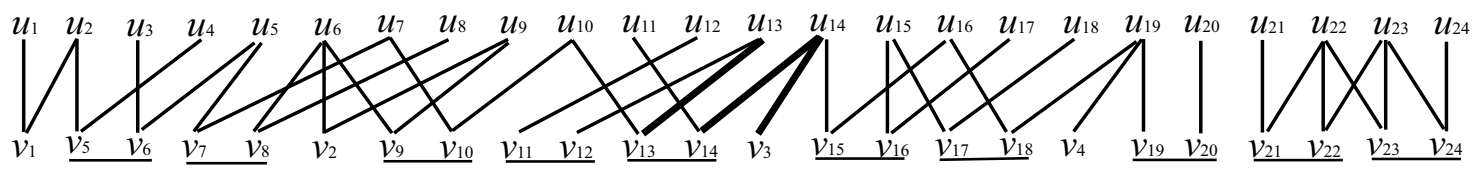

Figure 1: An example of $G$. The three bold edges $\left(u_{13}, v_{13}\right),\left(u_{14}, v_{14}\right)$, and $\left(u_{14}, v_{3}\right)$ each have weight 3 , and the other edges each have weight 1 . Also, for each $v_{j} \in \mathcal{J}_{2}, v_{j}$ and $v_{j+1}$ are underlined together. In other words, $n_{1}=4$.

A constrained matching of $G$ is a matching $M$ of $G$ such that for every $u_{i} \in U$ and every $v_{j} \in \mathcal{J}_{2},\left(u_{i}, v_{j}\right) \in M$ if and only if $\left(u_{i+1}, v_{j+1}\right) \in M$. The objective of 2-ISP is equivalent to finding a maximum-weight constrained matching in $G$. For each edge $\left(u_{i}, v_{j}\right)$ of $G$, let $w\left(u_{i}, v_{j}\right)$ denote the weight of the edge. For convenience, let $w\left(u_{i}, v_{j}\right)=0$ for all $\left(u_{i}, v_{j}\right) \notin E$. For a (constrained or unconstrained) matching $M$ of $G$, let $w_{1}(M)$ (respectively, $w_{2}(M)$ ) denote the total weight of edges $\left(u_{i}, v_{j}\right) \in M$ with $v_{j} \in \mathcal{J}_{1}$ (respectively, $v_{j} \in \mathcal{J}_{2} \cup \mathcal{J}_{2}^{\prime}$ ); let $w(M)=w_{1}(M)+w_{2}(M)$.

\footnotetext{
${ }^{2}$ For unweighted ISP where the profit of executing a job at each specific time interval is either 0 or 1 (independent of the job's length), Chuzhoy et al. [5] gave a 1.582-approximation algorithm. In this paper, our interest is in the weighted problem.

${ }^{3}$ The program is available to the public upon request to the authors.

${ }^{4}$ This corresponds to a simplified situation in NMR peak assignment, where each spin system has a few equally preferred matching segments of amino acids.

${ }^{5} \mathrm{~A}$ simple modification of this algorithm leads to a $(1.5+\epsilon)$-approximation algorithm for unweighted 2-ISP.
} 
Let $M^{*}$ be a maximum-weight constrained matching in $G$. In Sections $2.1,2.3$ through 2.5, we will design four algorithms each outputting a constrained matching in $G$. We will try to find a large constant $\epsilon$ such that the heaviest one among the four output matchings is of weight at least $\left(\frac{1}{2}+\epsilon\right) w\left(M^{*}\right)$. It will turn out that $\epsilon=\frac{1}{26}$. So, fix $\epsilon=\frac{1}{26}$ for the discussions in the rest of this section.

\subsection{Algorithm 1}

This algorithm will output a constrained matching of large weight when $w_{2}\left(M^{*}\right)$ is relatively large compared with $w_{1}\left(M^{*}\right)$. We first explain the idea behind the algorithm. Suppose that we partition the time interval $\mathcal{I}$ into shorter intervals, called basic intervals, in such a way that each basic interval, except possibly the first and the last (which may possibly consist of 1 or 2 time units), consists of 3 time units. There are exactly three such partitions of $\mathcal{I}$ (see Figure 2). Denote them by $P_{0}, P_{1}$, and $P_{2}$, respectively. With respect to each $P_{h}$ with $0 \leq h \leq 2$, consider the problem $\mathcal{Q}_{h}$ of finding a constrained scheduling which maximizes the total profit of the executed jobs, but subject to the constraint that each basic interval in $P_{h}$ can be assigned to at most one job and each executed job should be completed within a single basic interval in $P_{h}$. It is not so hard to see that each problem $\mathcal{Q}_{h}$ requires the computation of a maximum-weight (unconstrained) matching in a suitably constructed bipartite graph, and hence can be solved in polynomial time.

\begin{tabular}{|c|c|}
\hline$\tau$ & 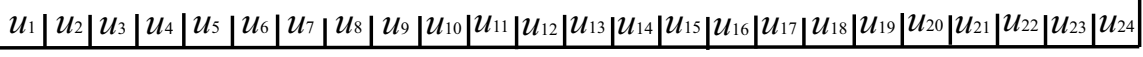 \\
\hline & \begin{tabular}{|lll|lll|lll|lll|lll|lll|lll|llll}
$u_{1}$ & $u_{2}$ & $u_{3}$ & $u_{4}$ & $u_{5}$ & $u_{6}$ & $u_{7}$ & $u_{8}$ & $u_{9}$ & $u_{10}$ & $u_{11}$ & $u_{12}$ & $u_{13}$ & $u_{14}$ & $u_{15}$ & $u_{16}$ & $u_{17}$ & $u_{18}$ & $u_{19}$ & $u_{20}$ & $u_{21}$ & $u_{22}$ & $u_{23}$ & $u_{24}$ \\
\end{tabular} \\
\hline & \begin{tabular}{l|lll|lll|lll|lll|lll}
$u_{1}$ & $u_{2}$ & $u_{3}$ & $u_{4}$ & $u_{5}$ & $u_{6}$ & $u_{7}$ & $u_{8}$ & $u_{9}$ & $u_{10}$ & $u_{11}$ & $u_{12}$ & $u_{13} \mid$ & $u_{14}$ & $u_{15}$ & $u_{16}$
\end{tabular} \mid $u_{1}$ \\
\hline & \begin{tabular}{l|lll|lll|lll|l}
$u_{2}$ & $u_{3}$ & $u_{4}$ & $u_{5}$ & $u_{6}$ & $u_{7}$ & $u_{8}$ & $u_{9}$ & $u_{10}$ & $u_{11}$ & $u_{12} u$ \\
\end{tabular} \\
\hline
\end{tabular}

Figure 2: An example of interval $\mathcal{I}$ and its partitions.

We claim that among the three problems $\mathcal{Q}_{h}$, the best one gives a scheduling by which the executed jobs achieve at least a total profit of $\frac{1}{3} w_{1}\left(M^{*}\right)+\frac{2}{3} w_{2}\left(M^{*}\right)$. This claim is actually easier to see, if we refer to a more constrained scheduling problem $\mathcal{Q}_{h}^{\prime}$ than $\mathcal{Q}_{h}$ by adding the following constraint:

- For each job $v_{j} \in \mathcal{J}_{1}$ and for each basic interval $b$ in $P_{h}$, only the primary time unit of $b$ can be assigned to $v_{j}$, where the primary time unit of $b$, is $u_{i}$ if $b$ consists of three time units $u_{i-1} u_{i} u_{i+1}$, is $u_{1}$ if $b$ consists of the first two time units $u_{1} u_{2}$ of $\mathcal{I}$, is $u_{I}$ if $b$ consists of the last two time units $u_{I-1} u_{I}$ of $\mathcal{I}$, is $b$ itself if $b$ consists of one time unit only.

[Comment: The crux is that for each basic interval $b$ consisting of at least two time units, each sub-interval of $b$ consisting of two time units must contain the primary time unit of $b$. Thus, by this constraint, we are allowed to assign at most one job to each basic interval. In turn, $\mathcal{Q}_{h}^{\prime}$ is more constrained than $\mathcal{Q}_{h}$.]

Consider an optimal (unconstrained) scheduling $M^{*}$. For each job $v_{j} \in \mathcal{J}_{2}$, if $M^{*}$ assigns $v_{j}$ to two time units $u_{i} u_{i+1}$, then this assignment of $v_{j}$ is also valid in exactly two problems among $\mathcal{Q}_{0}^{\prime}$, $\mathcal{Q}_{1}^{\prime}$, and $\mathcal{Q}_{2}^{\prime}$, because there are exactly two indices $h \in\{0,1,2\}$ such that some basic interval in $P_{h}$ contains both time units $u_{i} u_{i+1}$. Similarly, for each job $v_{j} \in \mathcal{J}_{1}$, if $M^{*}$ assigns $v_{j}$ to one time unit $u_{i}$, then this assignment of $v_{j}$ is also valid in at least one problem among $\mathcal{Q}_{0}^{\prime}, \mathcal{Q}_{1}^{\prime}$, and $\mathcal{Q}_{2}^{\prime}$, because there is at least one index $h \in\{0,1,2\}$ such that $u_{i}$ is the primary time unit of some basic interval in $P_{h}$. Thus, by inheriting from the optimal scheduling $M^{*}$, the three problems $\mathcal{Q}_{h}^{\prime}$ have moreconstrained schedulings $M_{h}^{*}$ such that $M_{h}^{*}$ is a sub-scheduling of $M^{*}$ and the three schedulings $M_{h}^{*}$ altogether achieve at least a total profit of $w_{1}\left(M^{*}\right)+2 w_{2}\left(M^{*}\right)$. Hence, the best more-constrained 
scheduling among $M_{1}^{*}, M_{2}^{*}$, and $M_{3}^{*}$ achieves at least a total profit of $\frac{1}{3} w_{1}\left(M^{*}\right)+\frac{2}{3} w_{2}\left(M^{*}\right)$. Indeed, we can prove the following better bound which is needed in later sections:

The best more-constrained scheduling among $M_{1}^{*}, M_{2}^{*}$, and $M_{3}^{*}$ achieves a total profit of at least $\frac{1}{3} w_{1}\left(M^{*}\right)+\frac{2}{3} w_{2}\left(M^{*}\right)+\frac{1}{3}\left(p_{1}+p_{I}\right)$, where $p_{1}=0$ (respectively, $\left.p_{I}=0\right)$ if $M^{*}$ assigns no job in $\mathcal{J}_{1}$ to $u_{1}$ (respectively, $u_{I}$ ), while $p_{1}$ (respectively, $p_{I}$ ) equals the weight of the edge of $M^{*}$ incident to $u_{1}$ (respectively, $u_{I}$ ) otherwise.

To see why we have this better bound, first note that there are exactly two indices $h \in\{0,1,2\}$ such that $u_{1}$ is the primary time unit of a basic interval in $P_{h}$. Similarly, there are exactly two indices $h \in\{0,1,2\}$ such that $u_{I}$ is the primary time unit of a basic interval in $P_{h}$. By these two facts, the better bound follows.

As it should be expected, the constrained scheduling problems $\mathcal{Q}_{h}$ may often lead to better experimental results than the more-constrained scheduling problems $\mathcal{Q}_{h}^{\prime}$. However, as for general theoretical results, we don't know if there is a difference between the two types of problems. Moreover, $\mathcal{Q}_{h}^{\prime}$ can be solved more efficiently than $\mathcal{Q}_{h}$. Hence, for simplicity, in the following exposition we will consider only the more-constrained scheduling problems $\mathcal{Q}_{h}^{\prime}$.

It is not hard to see that each more-constrained scheduling problem $\mathcal{Q}_{h}^{\prime}$ requires the computation of a maximum-weight (unconstrained) matching in a suitably constructed bipartite graph $G_{h}$, and hence can be solved in polynomial time. For clarity, we detail the construction of the graphs $G_{h}$ below.

For each index $h \in\{0,1,2\}$, let $G_{h}$ be the edge-weighted bipartite graph obtained from $G$ as follows: (See Figure 3 for an example of $G_{2}$ constructed from graph $G$ in Figure 1.)

1. For every $v_{j} \in \mathcal{J}_{2}$, merge the two vertices $v_{j}$ and $v_{j+1}$ into a single super-vertex $s_{j, j+1}$ (with all resulting multiple edges deleted).

2. For all $i$ such that $h+1 \leq i \leq I-2$ and $i-1 \equiv h(\bmod 3)$, perform the following three sub-steps:

(a) Merge $u_{i}, u_{i+1}$, and $u_{i+2}$ into a single super-vertex $t_{i, i+1, i+2}$ (with all resulting multiple edges deleted).

(b) For every $v_{j} \in \mathcal{J}_{1}$ that is a neighbor of $t_{i, i+1, i+2}$, if edge $\left(u_{i+1}, v_{j}\right)$ is not in the original input graph, then delete the edge between $t_{i, i+1, i+2}$ and $v_{j}$; otherwise, assign a weight of $w\left(u_{i+1}, v_{j}\right)$ to the edge between $t_{i, i+1, i+2}$ and $v_{j}$.

(c) For every $v_{j} \in \mathcal{J}_{2}$ such that $s_{j, j+1}$ is a neighbor of $t_{i, i+1, i+2}$, if neither $\left\{\left(u_{i}, v_{j}\right),\left(u_{i+1}, v_{j+1}\right)\right\}$ nor $\left\{\left(u_{i+1}, v_{j}\right),\left(u_{i+2}, v_{j+1}\right)\right\}$ is a matching in the original input graph, then delete the edge between $t_{i, i+1, i+2}$ and $s_{j, j+1}$; otherwise, assign a weight of $\max \left\{w\left(u_{i}, v_{j}\right)+\right.$ $\left.w\left(u_{i+1}, v_{j+1}\right), w\left(u_{i+1}, v_{j}\right)+w\left(u_{i+2}, v_{j+1}\right)\right\}$ to the edge between $t_{i, i+1, i+2}$ and $s_{j, j+1}$.

3. If neither $u_{1}$ nor $u_{2}$ was merged in Step 2a, then perform the following three sub-steps:

(a) Merge $u_{1}$ and $u_{2}$ into a single super-vertex $t_{1,2}$ (with all resulting multiple edges deleted).

(b) For every $v_{j} \in \mathcal{J}_{1}$ that is a neighbor of $t_{1,2}$, if edge $\left(u_{1}, v_{j}\right)$ is not in the original input graph, then delete the edge between $t_{1,2}$ and $v_{j}$; otherwise, assign a weight of $w\left(u_{1}, v_{j}\right)$ to the edge between $t_{1,2}$ and $v_{j}$.

(c) For every $v_{j} \in \mathcal{J}_{2}$ such that $s_{j, j+1}$ is a neighbor of $t_{1,2}$, if $\left\{\left(u_{1}, v_{j}\right),\left(u_{2}, v_{j+1}\right)\right\}$ is not a matching in the original input graph, then delete the edge between $t_{1,2}$ and $s_{j, j+1}$; otherwise, assign a weight of $w\left(u_{1}, v_{j}\right)+w\left(u_{2}, v_{j+1}\right)$ to the edge between $t_{1,2}$ and $s_{j, j+1}$.

4. If neither $u_{I-1}$ nor $u_{I}$ was merged in Step 2a, then perform the following three sub-steps:

(a) Merge $u_{I-1}$ and $u_{I}$ into a single super-vertex $t_{I-1, I}$ (with all resulting multiple edges deleted).

(b) For every $v_{j} \in \mathcal{J}_{1}$ that is a neighbor of $t_{I-1, I}$, if edge $\left(u_{I}, v_{j}\right)$ is not in the original input graph, then delete the edge between $t_{I-1, I}$ and $v_{j}$; otherwise, assign a weight of $w\left(u_{I}, v_{j}\right)$ to the edge between $t_{I-1, I}$ and $v_{j}$. 
(c) For every $v_{j} \in \mathcal{J}_{2}$ such that $s_{j, j+1}$ is a neighbor of $t_{I-1, I}$, if $\left\{\left(u_{I-1}, v_{j}\right),\left(u_{I}, v_{j+1}\right)\right\}$ is not a matching in the original input graph, then delete the edge between $t_{I-1, I}$ and $s_{j, j+1}$; otherwise, assign a weight of $w\left(u_{I-1}, v_{j}\right)+w\left(u_{I}, v_{j+1}\right)$ to the edge between $t_{I-1, I}$ and $s_{j, j+1}$.

5. If $u_{1}$ was merged in neither Step 2a nor Step 3a, then for every $v_{j} \in \mathcal{J}_{2}$ such that $s_{j, j+1}$ is a neighbor of $u_{1}$, delete the edge between $u_{1}$ and $s_{j, j+1}$.

6. If $u_{I}$ was merged in neither Step 2a nor Step 4a, then for every $v_{j} \in \mathcal{J}_{2}$ such that $s_{j, j+1}$ is a neighbor of $u_{I}$, delete the edge between $u_{I}$ and $s_{j, j+1}$.

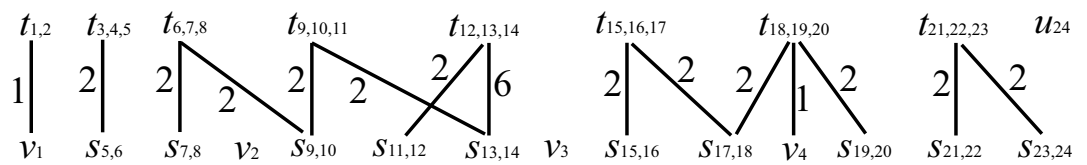

Figure 3: Graph $G_{2}$ constructed from graph $G$ in Figure 1. The number beside each edge is the weight of that edge.

For each $h \in\{0,1,2\}$, let $M_{h}$ be a maximum-weight matching in $G_{h}$. In our example (cf. Figure 3), $M_{2}$ may consist of the following edges: $\left(t_{1,2}, v_{1}\right),\left(t_{3,4,5}, s_{5,6}\right),\left(t_{6,7,8}, s_{7,8}\right),\left(t_{9,10,11}, s_{9,10}\right)$, $\left(t_{12,13,14}, s_{13,14}\right),\left(t_{15,16,17}, s_{15,16}\right),\left(t_{18,19,20}, s_{19,20}\right)$, and $\left(t_{21,22,23}, s_{21,22}\right)$. From each $M_{h}$, we can obtain a constrained matching $\bar{M}_{h}$ in the original input graph by performing the following steps in turn:

- Initialize $\bar{M}_{h}=\emptyset$.

- For each edge $\left(u_{i}, v_{j}\right) \in M_{h}$, add $\left(u_{i}, v_{j}\right)$ to $\bar{M}_{h}$.

- For each edge $\left(t_{i, i+1, i+2}, v_{j}\right) \in M_{h}$, add $\left(u_{i+1}, v_{j}\right)$ to $\bar{M}_{h}$.

- For each edge $\left(t_{1,2}, v_{j}\right) \in M_{h}$, add $\left(u_{1}, v_{j}\right)$ to $\bar{M}_{h}$.

- For each edge $\left(t_{I-1, I}, v_{j}\right) \in M_{h}$, add $\left(u_{I}, v_{j}\right)$ to $\bar{M}_{h}$.

- For each edge $\left(t_{i, i+1, i+2}, s_{j, j+1}\right) \in M_{h}$, if $w\left(u_{i}, v_{j}\right)+w\left(u_{i+1}, v_{j+1}\right) \geq w\left(u_{i+1}, v_{j}\right)+w\left(u_{i+2}, v_{j+1}\right)$, then add edges $\left(u_{i}, v_{j}\right)$ and $\left(u_{i+1}, v_{j+1}\right)$ to $\bar{M}_{h}$; otherwise, add edges $\left(u_{i+1}, v_{j}\right)$ and $\left(u_{i+2}, v_{j+1}\right)$ to $\bar{M}_{h}$.

- For each edge $\left(t_{1,2}, s_{j, j+1}\right) \in M_{h}$, add edges $\left(u_{1}, v_{j}\right)$ and $\left(u_{2}, v_{j+1}\right)$ to $\bar{M}_{h}$.

- For each edge $\left(t_{I-1, I}, s_{j, j+1}\right) \in M_{h}$, add $\left(u_{I-1}, v_{j}\right)$ and $\left(u_{I}, v_{j+1}\right)$ to $\bar{M}_{h}$.

Note that $w\left(\bar{M}_{h}\right)=w\left(M_{h}\right)$. In our example (cf. Figures 1 and 3), if $M_{2}$ is as mentioned above, then $\bar{M}_{2}$ consists of the following edges: $\left(u_{1}, v_{1}\right),\left(u_{4}, v_{5}\right),\left(u_{5}, v_{6}\right),\left(u_{7}, v_{7}\right),\left(u_{8}, v_{8}\right),\left(u_{9}, v_{9}\right)$, $\left(u_{10}, v_{10}\right),\left(u_{13}, v_{13}\right),\left(u_{14}, v_{14}\right),\left(u_{15}, v_{16}\right),\left(u_{16}, v_{17}\right),\left(u_{19}, v_{19}\right),\left(u_{20}, v_{20}\right),\left(u_{21}, v_{21}\right)$, and $\left(u_{22}, v_{22}\right)$.

In summary, we have established the following lemma:

Lemma 2.1 A constrained matching $Z_{1}$ in $G$ can be found in $O\left(I\left(n_{1}+n_{2}\right)\left(I+n_{1}+n_{2}\right)\right)$ time, whose weight is at least $\frac{1}{3} w_{1}\left(M^{*}\right)+\frac{2}{3} w_{2}\left(M^{*}\right)+\frac{1}{3}\left(p_{1}+p_{I}\right)$, where $p_{1}=0$ (respectively, $\left.p_{I}=0\right)$ if $u_{1}$ (respectively, $u_{I}$ ) is not matched to a vertex of $\mathcal{J}_{1}$ by $M^{*}$, while $p_{1}$ (respectively, $p_{I}$ ) equals the weight of the edge of $M^{*}$ incident to $u_{1}$ (respectively, $u_{I}$ ) otherwise.

Corollary 2.2 If $w_{1}\left(M^{*}\right) \leq\left(\frac{1}{2}-3 \epsilon\right) w\left(M^{*}\right)$, then $w\left(Z_{1}\right) \geq\left(\frac{1}{2}+\epsilon\right) w\left(M^{*}\right)$.

Proof. Assume $w_{1}\left(M^{*}\right) \leq\left(\frac{1}{2}-3 \epsilon\right) w\left(M^{*}\right)$. Then, $w_{2}\left(M^{*}\right)=w\left(M^{*}\right)-w_{1}\left(M^{*}\right) \geq\left(\frac{1}{2}+3 \epsilon\right) w\left(M^{*}\right)$. Moreover, by Lemma 2.1, $w\left(Z_{1}\right) \geq \frac{1}{3} w_{1}\left(M^{*}\right)+\frac{2}{3} w_{2}\left(M^{*}\right)=\frac{1}{3} w\left(M^{*}\right)+\frac{1}{3} w_{2}\left(M^{*}\right)$. Thus, $w\left(Z_{1}\right) \geq$ $\left(\frac{1}{3}+\frac{1}{3}\left(\frac{1}{2}+3 \epsilon\right)\right) w\left(M^{*}\right) \geq\left(\frac{1}{2}+\epsilon\right) w\left(M^{*}\right)$. 


\subsection{Preparing for the other three algorithms}

Before running the other three algorithms, we need to compute a maximum-weight unconstrained matching $M_{\mathrm{un}}^{*}$ of $G$. The unconstrained matching $M_{\mathrm{un}}^{*}$ will be an additional input to the other three algorithms. Therefore, before proceeding to the details of the algorithms, fix a maximumweight unconstrained matching $M_{\mathrm{un}}^{*}$ of $G$. See Figure 4 for an example. The algorithms in Sec-

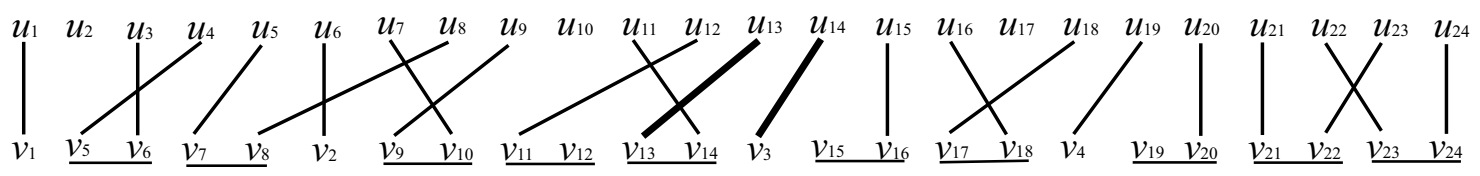

Figure 4: A maximum-weight unconstrained matching $M_{\mathrm{un}}^{*}$ of the graph $G$ in Figure 1.

tions 2.3 through 2.5 will use $M_{\mathrm{un}}^{*}$ in a sophisticated way. But first, we use $M_{\mathrm{un}}^{*}$ to define several subsets of $U$ as follows.

- $U_{0}=\left\{u_{i} \in U \mid u_{i}\right.$ is not matched by $\left.M_{\mathrm{un}}^{*}\right\}$.

- $U_{1}=\left\{u_{i} \in U \mid u_{i}\right.$ is matched to a $v_{j} \in \mathcal{J}_{1}$ by $\left.M_{\mathrm{un}}^{*}\right\}$.

- $U_{2,1}=\left\{u_{i} \in U \mid u_{i}\right.$ is matched to a $v_{j} \in \mathcal{J}_{2}$ by $\left.M_{\mathrm{un}}^{*}\right\}$.

- $U_{2,2}=\left\{u_{i} \in U \mid u_{i}\right.$ is matched to a $v_{j} \in \mathcal{J}_{2}^{\prime}$ by $\left.M_{\mathrm{un}}^{*}\right\}$.

- $W=\left\{u_{i} \in U_{1} \mid u_{i-1} \in U_{2,1}\right.$ and $\left.u_{i+1} \in U_{2,2}\right\}$.

- $W_{L}=\left\{u_{i} \in U \mid u_{i+1} \in W\right\}$ and $W_{R}=\left\{u_{i} \in U \mid u_{i-1} \in W\right\}$.

In our example (cf. Figures 1 and 4 ), $U_{0}=\left\{u_{2}, u_{10}, u_{17}\right\}, U_{1}=\left\{u_{1}, u_{6}, u_{14}, u_{19}\right\}, U_{2,1}=$ $\left\{u_{4}, u_{5}, u_{9}, u_{12}, u_{13}, u_{18}, u_{21}, u_{23}\right\}, U_{2,2}=\left\{u_{3}, u_{7}, u_{8}, u_{11}, u_{15}, u_{16}, u_{20}, u_{23}, u_{24}\right\}, W=\left\{u_{6}, u_{14}, u_{19}\right\}$, $W_{L}=\left\{u_{5}, u_{13}, u_{18}\right\}$, and $W_{R}=\left\{u_{7}, u_{15}, u_{20}\right\}$. In general, whenever $u_{i} \in W$, we have $u_{i-1} \in W_{L}$ and $u_{i+1} \in W_{R}$. Moreover, since $W \subseteq U_{1}, W_{L} \subseteq U_{2,1}$, and $W_{R} \subseteq U_{2,2}$, no two sets among $W, W_{L}$ and $W_{R}$ can intersect.

A common idea behind the forthcoming algorithms is to divide the weights $w_{1}\left(M^{*}\right)$ and $w_{2}\left(M^{*}\right)$ into smaller parts, based on the aforementioned subsets of $U$. The smaller parts are defined as follows.

- $\beta_{L}$ is the total weight of all edges $\left(u_{i}, v_{j}\right) \in M^{*}$ such that $u_{i} \in W_{L}$ and $v_{j} \in \mathcal{J}_{1}$.

- $\beta$ is the total weight of all edges $\left(u_{i}, v_{j}\right) \in M^{*}$ such that $u_{i} \in W$ and $v_{j} \in \mathcal{J}_{1}$.

- $\beta_{R}$ is the total weight of all edges $\left(u_{i}, v_{j}\right) \in M^{*}$ such that $u_{i} \in W_{R}$ and $v_{j} \in \mathcal{J}_{1}$.

- $\bar{\beta}=w_{1}\left(M^{*}\right)-\beta_{L}-\beta-\beta_{R}$.

- $\alpha_{0}$ is the total weight of all edges $\left(u_{i}, v_{j}\right) \in M^{*}$ such that either $v_{j} \in \mathcal{J}_{2}$ and $\left\{u_{i}, u_{i+1}\right\} \cap W=\emptyset$, or $v_{j} \in \mathcal{J}_{2}^{\prime}$ and $\left\{u_{i-1}, u_{i}\right\} \cap W=\emptyset$.

- $\alpha_{1}$ is the total weight of all edges $\left(u_{i}, v_{j}\right) \in M^{*}$ such that either $v_{j} \in \mathcal{J}_{2}$ and $\left\{u_{i}, u_{i+1}\right\} \cap W \neq \emptyset$, or $v_{j} \in \mathcal{J}_{2}^{\prime}$ and $\left\{u_{i-1}, u_{i}\right\} \cap W \neq \emptyset$.

Lemma 2.3 $\alpha_{0}+\alpha_{1}=w_{2}\left(M^{*}\right)$ and $\beta_{L}+\beta+\beta_{R}+\bar{\beta}=w_{1}\left(M^{*}\right)$.

Proof. Obvious.

Now, we are ready to explain how the four algorithms are related. The algorithm in Section 2.3 , called Algorithm 2, will output a constrained matching of weight at least $\frac{1}{3} \bar{\beta}+\frac{2}{3} \alpha_{0}+\beta+\frac{2}{3}\left(\beta_{L}+\beta_{R}\right)$. 
The algorithm in Section 2.4, called Algorithm 3, will output a constrained matching of weight at least $\beta+\bar{\beta}+\alpha_{1}$. Thus, if $\beta \geq\left(\frac{1}{6}+\frac{5}{3} \epsilon\right) w\left(M^{*}\right)$, then Algorithm 2 or 3 will output a constrained matching of weight at least $\left(\frac{1}{2}+\epsilon\right) w\left(M^{*}\right)$ (see Corollary 2.6 below). On the other hand, if $\beta<$ $\left(\frac{1}{6}+\frac{5}{3} \epsilon\right) w\left(M^{*}\right)$, then Algorithm 1 or 4 will output a constrained matching of weight at least $\left(\frac{1}{2}+\epsilon\right) w\left(M^{*}\right)$ (see Section 2.6).

\subsection{Algorithm 2}

We first explain the idea behind the algorithm. The removal of the vertices in $W$ leaves $|W|+1$ blocks of $U$ each of which consists of consecutive vertices of $U$. For each block $b$, we use the idea of Algorithm 1 to construct three graphs $G_{b, 0}, G_{b, 1}, G_{b, 2}$. For each $h \in\{0,1,2\}$, we consider the graph $\cup_{b} G_{b, h}$ where $b$ ranges over all blocks, and obtain a new graph $G_{h}^{\prime}$ from $\cup_{b} G_{b, h}$ by adding the vertices of $W$ and the edges $\left\{u_{i}, v_{j}\right\}$ of $G$ such that $u_{i} \in W$ and $v_{j} \in \mathcal{J}_{1}$. We then compute a maximumweight (unconstrained) matching in each $G_{h}^{\prime}$, and further convert it to a constrained matching $\bar{M}_{h}^{\prime}$ of $G$ as in Algorithm 1. The output of Algorithm 2 is the heaviest matching among $\bar{M}_{0}^{\prime}, \bar{M}_{1}^{\prime}, \bar{M}_{2}^{\prime}$. In our example (cf. Figures 1 and 4$), G_{2}^{\prime}$ is as shown in Figure 5, and $\bar{M}_{2}^{\prime}$ may consist of the following edges: $\left(u_{1}, v_{1}\right),\left(u_{4}, v_{5}\right),\left(u_{5}, v_{6}\right),\left(u_{6}, v_{2}\right),\left(u_{7}, v_{7}\right),\left(u_{8}, v_{8}\right),\left(u_{9}, v_{9}\right),\left(u_{10}, v_{10}\right),\left(u_{12}, v_{11}\right),\left(u_{13}, v_{12}\right)$, $\left(u_{14}, v_{3}\right),\left(u_{15}, v_{17}\right),\left(u_{16}, v_{18}\right),\left(u_{19}, v_{4}\right),\left(u_{22}, v_{21}\right)$, and $\left(u_{23}, v_{22}\right)$.

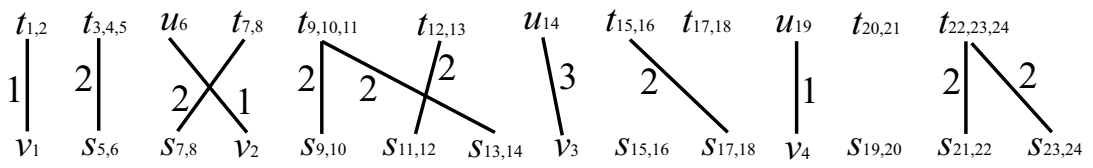

Figure 5: Graph $G_{2}^{\prime}$ constructed from graph $G$ in Figure 1 and matching $M_{\text {un }}^{*}$ in Figure 4 . The number beside each edge is the weight of that edge.

We next proceed to the details of Algorithm 2. Recall that the removal of the vertices in $W$ leaves $|W|+1$ blocks of $U$ each of which consists of consecutive vertices of $U$. For each block $b$, let $G_{b}$ be the subgraph of $G$ induced by $V \cup\left\{u_{i} \in U \mid u_{i}\right.$ is a vertex in block $\left.b\right\}$.

1. For each block $b$, perform the following steps.

(a) Delete all edges $\left\{u_{i}, v_{j}\right\}$ from $G_{b}$ such that $u_{i}$ is the first vertex in block $b$ and $v_{j} \in \mathcal{J}_{2}^{\prime}$; further delete all edges $\left\{u_{i}, v_{j}\right\}$ from $G_{b}$ such that $u_{i}$ is the last vertex in block $b$ and $v_{j} \in \mathcal{J}_{2}$.

(b) Construct three edge-weighted bipartite graphs $G_{b, 0}, G_{b, 1}, G_{b, 2}$ from $G_{b}$ in the same way as Algorithm 1 constructs the graphs $G_{0}, G_{1}, G_{2}$ from $G$.

2. For each $h \in\{0,1,2\}$, construct a new edge-weighted bipartite graph $G_{h}^{\prime}$ as follows. The vertex set of $G_{h}^{\prime}$ is the union of $W$ and the vertex sets of the graphs $G_{b, h}$ where $b$ ranges over all blocks. Note that even if a vertex appears in two or more of the graphs $G_{b, h}$, it appears in $G_{h}^{\prime}$ only once. The edges of the graphs $G_{b, h}$ where $b$ ranges over all blocks are also edges in $G_{h}^{\prime}$ and inherit their weights to $G_{h}^{\prime}$. Moreover, each edge $\left(u_{i}, v_{j}\right)$ in $G$ such that $u_{i} \in W$ and $v_{j} \in \mathcal{J}_{1}$ is also an edge in $G_{h}^{\prime}$ and inherits its weight from $G$ to $G_{h}^{\prime}$. $G_{h}^{\prime}$ has no other edges.

3. For each $h \in\{0,1,2\}$, compute a maximum-weight matching $M_{h}^{\prime}$ in $G_{h}^{\prime}$, and then compute a constrained matching $\bar{M}_{h}^{\prime}$ in $G$ from $M_{h}^{\prime}$ in the same way as Algorithm 1 computes the constrained matching $\bar{M}_{h}$ in $G$ from $M_{h}$.

4. Let $Z_{2}$ be the maximum-weight matching among the matchings $M_{0}^{\prime}, M_{1}^{\prime}, M_{2}^{\prime}$. Output $Z_{2}$.

Lemma $2.4 w\left(Z_{2}\right) \geq \frac{1}{3} \bar{\beta}+\frac{2}{3} \alpha_{0}+\beta+\frac{2}{3}\left(\beta_{L}+\beta_{R}\right)$.

Proof. Immediate from Lemma 2.1 and Algorithm 2. 


\subsection{Algorithm 3}

We first explain the idea behind Algorithm 3. Suppose that we partition the time interval $\mathcal{I}$ into shorter intervals in such a way that each shorter interval consists of either one time unit or three time units $u_{i-1} u_{i} u_{i+1}$ where $u_{i} \in W$. There is only one such partition of $\mathcal{I}$. Further suppose that we want to execute at most one job in each of the shorter intervals, while maximizing the total profit of the executed jobs. This problem can be solved in polynomial time by computing a maximum-weight (unconstrained) matching in a suitably constructed bipartite graph. We can prove that this matching results in a scheduling by which the executed jobs achieve at least a total profit of $\beta+\bar{\beta}+\alpha_{1}$.

We next proceed to the details of Algorithm 3. Algorithm 3 computes a constrained matching of $G$ as follows. (See Figure 6 for an example.)

1. Construct a new edge-weighted bipartite graph $G^{\prime \prime}$ from $G$ as follows:

(a) For each $u_{i} \in W$, merge $u_{i-1}, u_{i}$ and $u_{i+1}$ into a super-vertex $t_{i-1, i, i+1}$ (with all resulting multiple edges deleted).

(b) For each $v_{j} \in \mathcal{J}_{2}$, merge the two vertices $v_{j}$ and $v_{j+1}$ into a super-vertex $s_{j, j+1}$ (with all resulting multiple edges deleted).

(c) For each edge $\left(t_{i-1, i, i+1}, v_{j}\right)$ such that $v_{j} \in \mathcal{J}_{1}$, if $\left(u_{i}, v_{j}\right)$ is not an edge in the original input graph, then delete the edge $\left(t_{i-1, i, i+1}, v_{j}\right)$; otherwise, assign a weight of $w\left(u_{i}, v_{j}\right)$ to the edge $\left(t_{i-1, i, i+1}, v_{j}\right)$.

(d) For each edge $\left(t_{i-1, i, i+1}, s_{j, j+1}\right)$, if neither $\left\{\left(u_{i-1}, v_{j}\right),\left(u_{i}, v_{j+1}\right)\right\}$ nor $\left\{\left(u_{i}, v_{j}\right),\left(u_{i+1}, v_{j+1}\right)\right\}$ is a matching in the original input graph, then delete the edge $\left(t_{i-1, i, i+1}, s_{j, j+1}\right)$; otherwise, assign a weight of $\max \left\{w\left(u_{i-1}, v_{j}\right)+w\left(u_{i}, v_{j+1}\right), w\left(u_{i}, v_{j}\right)+w\left(u_{i+1}, v_{j+1}\right)\right\}$ to the edge $\left(t_{i-1, i, i+1}, s_{j, j+1}\right)$.

(e) Delete all edges $\left(u_{i}, s_{j, j+1}\right)$. (Note that $u_{i} \notin W_{L} \cup W \cup W_{R}$.)

2. Compute a maximum-weight unconstrained matching $M^{\prime \prime}$ in $G^{\prime \prime}$.

3. Construct a constrained matching $Z_{3}$ in $G$ from $M^{\prime \prime}$ as follows.

(a) Initialize $Z_{3}=\emptyset$.

(b) For each edge $\left(u_{i}, v_{j}\right) \in M^{\prime \prime}$, add $\left(u_{i}, v_{j}\right)$ to $Z_{3}$.

(c) For each edge $\left(t_{i-1, i, i+1}, v_{j}\right) \in M^{\prime \prime}$, add $\left(u_{i}, v_{j}\right)$ to $Z_{3}$.

(d) For each edge $\left(t_{i-1, i, i+1}, s_{j, j+1}\right) \in M^{\prime \prime}$, if $w\left(u_{i-1}, v_{j}\right)+w\left(u_{i}, v_{j+1}\right) \geq w\left(u_{i}, v_{j}\right)+w\left(u_{i+1}, v_{j+1}\right)$, then add edges $\left(u_{i-1}, v_{j}\right)$ and $\left(u_{i}, v_{j+1}\right)$ to $Z_{3}$; otherwise, add edges $\left(u_{i}, v_{j}\right)$ and $\left(u_{i+1}, v_{j+1}\right)$ to $Z_{3}$.

4. Output $Z_{3}$.

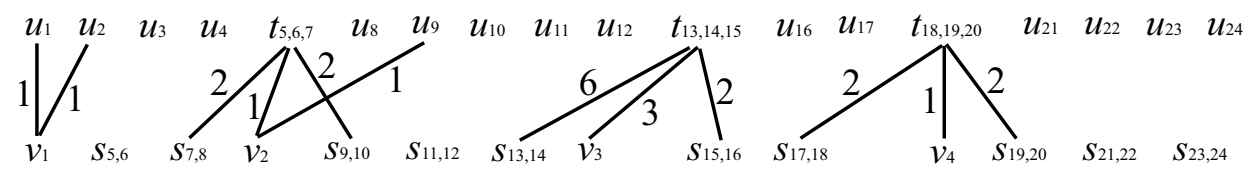

Figure 6: Graph $G^{\prime \prime}$ constructed from graph $G$ in Figure 1 and matching $M_{\text {un }}^{*}$ in Figure 4. The number beside each edge is the weight of that edge.

In our example (cf. Figures 1 and 6$), M^{\prime \prime}$ may consist of the following edges: $\left(u_{1}, v_{1}\right),\left(t_{5,6,7}, s_{7,8}\right)$, $\left(u_{9}, v_{2}\right),\left(t_{13,14,15}, s_{13,14}\right)$, and $\left(t_{18,19,20}, s_{17,18}\right)$, and in turn $Z_{3}$ consists of the following edges: $\left(u_{1}, v_{1}\right),\left(u_{5}, v_{7}\right),\left(u_{6}, v_{8}\right),\left(u_{9}, v_{2}\right),\left(u_{13}, v_{13}\right),\left(u_{14}, v_{14}\right),\left(u_{18}, v_{17}\right)$, and $\left(u_{19}, v_{18}\right)$. 
Lemma $2.5 w\left(Z_{3}\right) \geq \beta+\bar{\beta}+\alpha_{1}$.

Proof. Similar to the proof of Lemma 2.1.

Corollary 2.6 If $\beta \geq\left(\frac{1}{6}+\frac{5}{3} \epsilon\right) w\left(M^{*}\right)$, then $\max \left\{w\left(Z_{2}\right), w\left(Z_{3}\right)\right\} \geq\left(\frac{1}{2}+\epsilon\right) w\left(M^{*}\right)$.

Proof. It suffices to show that if $\max \left\{w\left(Z_{2}\right), w\left(Z_{3}\right)\right\}<\left(\frac{1}{2}+\epsilon\right) w\left(M^{*}\right)$, then $\beta<\left(\frac{1}{6}+\frac{5}{3} \epsilon\right) w\left(M^{*}\right)$. So, assume that $\max \left\{w\left(Z_{2}\right), w\left(Z_{3}\right)\right\}<\left(\frac{1}{2}+\epsilon\right) w\left(M^{*}\right)$. Then, we have the following two inequalities:

$$
\begin{aligned}
& w\left(Z_{2}\right)<\left(\frac{1}{2}+\epsilon\right) w\left(M^{*}\right) . \\
& w\left(Z_{3}\right)<\left(\frac{1}{2}+\epsilon\right) w\left(M^{*}\right) .
\end{aligned}
$$

Combining Inequality 2.1 and the inequality in Lemma 2.4, we obtain a new inequality, and further multiply it by a factor $\frac{3}{2}$ to obtain:

$$
\alpha_{0}+\frac{3}{2} \beta+\frac{1}{2} \bar{\beta}+\beta_{L}+\beta_{R}<\left(\frac{3}{4}+\frac{3}{2} \epsilon\right) w\left(M^{*}\right)
$$

Moreover, combining Inequality 2.2 and the inequality in Lemma 2.5, we obtain:

$$
\alpha_{1}+\beta+\bar{\beta}<\left(\frac{1}{2}+\epsilon\right) w\left(M^{*}\right)
$$

Now, adding Inequalities 2.3 and 2.4, we obtain

$$
\alpha_{0}+\alpha_{1}+\frac{5}{2} \beta+\frac{3}{2} \bar{\beta}+\beta_{L}+\beta_{R}<\left(\frac{5}{4}+\frac{5}{2} \epsilon\right) w\left(M^{*}\right) .
$$

In turn, by Lemma 2.3 , we have

$$
w_{1}\left(M^{*}\right)+w_{2}\left(M^{*}\right)+\frac{3}{2} \beta+\frac{1}{2} \bar{\beta}<\left(\frac{5}{4}+\frac{5}{2} \epsilon\right) w\left(M^{*}\right) .
$$

Using the fact that $w_{1}\left(M^{*}\right)+w_{2}\left(M^{*}\right)=w\left(M^{*}\right)$ and $\bar{\beta} \geq 0$, we finally obtain

$$
\frac{3}{2} \beta<\left(\frac{1}{4}+\frac{5}{2} \epsilon\right) w\left(M^{*}\right) \text {, or equivalently, } \beta<\left(\frac{1}{6}+\frac{5}{3} \epsilon\right) w\left(M^{*}\right),
$$

which completes the proof.

\subsection{Algorithm 4}

The idea behind Algorithm 4 is to convert $M_{\mathrm{un}}^{*}$ to a constrained matching of $G$. To convert $M_{\mathrm{un}}^{*}$, we partition $U_{1} \cup U_{2,1}$ (respectively, $U_{1} \cup U_{2,2}$ ) into two subsets none of which contains two vertices $u_{i}$ and $u_{i+1}$ such that $u_{i} \in U_{2,1}$ (respectively, $u_{i+1} \in U_{2,2}$ ). The set of edges of $M_{\text {un }}^{*}$ incident to the vertices of each such subset can be extended to a constrained matching of $G$. In this way, we obtain four constrained matchings of $G$. Algorithm 4 outputs the heaviest one among the four matchings. We can prove that the weight of the output matching is at least $w\left(M_{\mathrm{un}}^{*}\right) / 2$.

We next proceed to the details of Algorithm 4. Algorithm 4 computes a constrained matching in $G$ as follows. (See Figure 7 for an example.) 
1. Starting at $u_{1}$, divide $U$ into segments each of which is in the following form:

$$
u_{i-\ell} u_{i-\ell+1} \cdots u_{i-1} u_{i} u_{i+1} \cdots u_{i+r-1} u_{i+r},
$$

where $u_{j} \in U_{2,1}$ for all $i-\ell \leq j \leq i-1, u_{j} \in U_{2,2}$ for all $i+1 \leq j \leq i+r, u_{i-\ell-1} \notin U_{2,1}$, $u_{i+r+1} \notin U_{2,2}$, and $u_{i}$ has no restriction. Note that $\ell$ and/or $r$ may be equal to zero. We call $u_{i}$ the center of the segment. For each segment $s$, let $c(s)$ denote the integer $i$ such that $u_{i}$ is the center of $s$; let $\ell(s)$ denote the number of vertices in $s$ that precede $u_{c(s)}$; let $r(s)$ denote the number of vertices in $s$ that succeed $u_{c(s)}$.

[Comment: In our example (cf. Figure 7), $U$ is divided into 8 segments. We name them from left to right as $s_{1}, \ldots, s_{8}$. For example, $s_{1}$ consists of only $u_{1}$ while $s_{3}$ consists of $u_{4}, \ldots, u_{8}$. Moreover, $c\left(s_{1}\right)=1, \ell\left(s_{1}\right)=r\left(s_{1}\right)=0, c\left(s_{2}\right)=2, \ell\left(s_{2}\right)=0, r\left(s_{2}\right)=1, c\left(s_{3}\right)=6$, $\ell\left(s_{3}\right)=r\left(s_{3}\right)=2, c\left(s_{4}\right)=10, \ell\left(s_{4}\right)=r\left(s_{4}\right)=1, c\left(s_{5}\right)=14, \ell\left(s_{5}\right)=r\left(s_{5}\right)=2, c\left(s_{6}\right)=17$, $\ell\left(s_{6}\right)=r\left(s_{6}\right)=0, c\left(s_{7}\right)=19, \ell\left(s_{7}\right)=r\left(s_{7}\right)=1, c\left(s_{8}\right)=22, \ell\left(s_{8}\right)=1$, and $r\left(s_{8}\right)=2$. Alternatively, it is also valid that $c\left(s_{8}\right)=23, \ell\left(s_{8}\right)=2$, and $r\left(s_{8}\right)=1$.

Given $M_{\mathrm{un}}^{*}$ (and hence the partition of $U$ as $U_{0} \cup U_{1} \cup U_{2,1} \cup U_{2,2}$ ), the division of $U$ into segments is unique. To see this, consider two relations $\mathcal{R}_{\text {left }}$ and $\mathcal{R}_{\text {right }}$ defined on $U$ as follows: For every pair $\left(u_{i}, u_{j}\right), u_{i} \mathcal{R}_{\text {left }} u_{j}$ if and only if $j=i+1$ and $u_{i} \in U_{2,1} ; u_{i} \mathcal{R}_{\text {right }} u_{j}$ if and only if $j=i-1$ and $u_{i} \in U_{2,2}$. Then, the segments one-to-one correspond to the equivalence classes of the symmetric and transitive closure of the relation $\left.\mathcal{R}_{\text {left }} \cup \mathcal{R}_{\text {right }}.\right]$

2. For each segment $s$, compute two integers $x_{s}$ and $y_{s}$ as follows:

- If $u_{c(s)} \in U_{0}$, then $x_{s}=c(s)-1$ and $y_{s}=c(s)+1$.

- If $u_{c(s)} \in U_{1}$, then $x_{s}=y_{s}=c(s)$.

- If $u_{c(s)} \in U_{2,1}$, then $x_{s}=c(s)$ and $y_{s}=c(s)+1$.

- If $u_{c(s)} \in U_{2,2}$, then $x_{s}=c(s)-1$ and $y_{s}=c(s)$.

[Comment: In our example (cf. Figure 7), $x_{s_{1}}=y_{s_{1}}=1, x_{s_{2}}=1, y_{s_{2}}=3, x_{s_{3}}=y_{s_{3}}=6$, $x_{s_{4}}=9, y_{s_{4}}=11, x_{s_{5}}=y_{s_{5}}=14, x_{s_{6}}=16, y_{s_{6}}=18, x_{s_{7}}=y_{s_{7}}=19, x_{s_{8}}=22$, and $y_{s_{8}}=23$.

In other words, for each segment $s, u_{x_{s}}$ is the rightmost vertex in $s$ with $u_{x_{s}} \in U_{2,1} \cup U_{1}$, while $u_{y_{s}}$ is the leftmost vertex in $s$ with $u_{y_{s}} \in U_{2,2} \cup U_{1}$.]

3. Let

$$
\begin{aligned}
& U_{2,1}^{e}=\bigcup_{s}\left\{u_{i} \mid\left(x_{s}-i\right) \bmod 2=0, c(s)-\ell(s) \leq i \leq x_{s}\right\}, \\
& U_{2,1}^{o}=\bigcup_{s}\left\{u_{i} \mid\left(x_{s}-i\right) \bmod 2=1, c(s)-\ell(s) \leq i \leq x_{s}\right\}, \\
& U_{2,2}^{e}=\bigcup_{s}\left\{u_{i} \mid\left(i-y_{s}\right) \bmod 2=0, y_{s} \leq i \leq c(s)+r(s)\right\}, \\
& U_{2,2}^{o}=\bigcup_{s}\left\{u_{i} \mid\left(i-y_{s}\right) \bmod 2=1, y_{s} \leq i \leq c(s)+r(s)\right\},
\end{aligned}
$$

where $s$ runs over all segments.

[Comment: In our example (cf. Figure 7), $U_{2,1}^{e}=\left\{u_{1}, u_{4}, u_{6}, u_{9}, u_{12}, u_{14}, u_{19}, u_{22}\right\}, U_{2,1}^{o}=$ $\left\{u_{5}, u_{13}, u_{18}, u_{21}\right\}, U_{2,2}^{e}=\left\{u_{1}, u_{3}, u_{6}, u_{8}, u_{11}, u_{14}, u_{16}, u_{19}, u_{23}\right\}$, and $U_{2,2}^{o}=\left\{u_{7}, u_{15}, u_{20}, u_{24}\right\}$.

Note that if a vertex $u_{i} \in U$ belongs to more than one of the four sets $U_{2,1}^{e}, U_{2,1}^{o}, U_{2,2}^{e}, U_{2,2}^{o}$, then $u_{i} \in U_{1}, u_{i}$ is the center of the segment containing $u_{i}$, and $u_{i}$ belongs to only $U_{2,1}^{e}$ and $\left.U_{2,2}^{e} \cdot\right]$ 
4. Let

$$
\begin{aligned}
& M_{2,1}^{e}=\left\{\left(u_{i}, v_{j}\right) \in M_{\mathrm{un}}^{*} \mid u_{i} \in U_{2,1}^{e}\right\} \cup\left\{\left(u_{i+1}, v_{j+1}\right) \mid u_{i} \in U_{2,1}^{e} \cap U_{2,1} \text { and }\left\{u_{i}, v_{j}\right\} \in M_{\mathrm{un}}^{*}\right\}, \\
& M_{2,1}^{o}=\left\{\left(u_{i}, v_{j}\right) \in M_{\mathrm{un}}^{*} \mid u_{i} \in U_{2,1}^{o}\right\} \cup\left\{\left(u_{i+1}, v_{j+1}\right) \mid u_{i} \in U_{2,1}^{o} \cap U_{2,1} \text { and }\left\{u_{i}, v_{j}\right\} \in M_{\mathrm{un}}^{*}\right\}, \\
& M_{2,2}^{e}=\left\{\left(u_{i}, v_{j}\right) \in M_{\mathrm{un}}^{*} \mid u_{i} \in U_{2,2}^{e}\right\} \cup\left\{\left(u_{i-1}, v_{j-1}\right) \mid u_{i} \in U_{2,2}^{e} \cap U_{2,2} \text { and }\left\{u_{i}, v_{j}\right\} \in M_{\mathrm{un}}^{*}\right\}, \\
& M_{2,2}^{o}=\left\{\left(u_{i}, v_{j}\right) \in M_{\mathrm{un}}^{*} \mid u_{i} \in U_{2,2}^{o}\right\} \cup\left\{\left(u_{i-1}, v_{j-1}\right) \mid u_{i} \in U_{2,2}^{o} \cap U_{2,2} \text { and }\left\{u_{i}, v_{j}\right\} \in M_{\mathrm{un}}^{*}\right\} .
\end{aligned}
$$

[Comment: $M_{2,1}^{e}, M_{2,1}^{o}, M_{2,2}^{e}, M_{2,2}^{o}$ are constrained matchings in $G$ (cf. Lemma 2.7). In our example (cf. Figure 7), the edges in $M_{2,1}^{e}$ are $\left(u_{1}, v_{1}\right),\left(u_{4}, v_{5}\right),\left(u_{5}, v_{6}\right),\left(u_{6}, v_{2}\right),\left(u_{9}, v_{9}\right)$, $\left(u_{10}, v_{10}\right),\left(u_{12}, v_{11}\right),\left(u_{13}, v_{12}\right),\left(u_{14}, v_{3}\right),\left(u_{19}, v_{4}\right),\left(u_{22}, v_{23}\right)$, and $\left(u_{23}, v_{24}\right)$; the edges in $M_{2,1}^{o}$ are $\left(u_{5}, v_{7}\right),\left(u_{6}, v_{8}\right),\left(u_{13}, v_{13}\right),\left(u_{14}, v_{14}\right),\left(u_{18}, v_{17}\right),\left(u_{19}, v_{18}\right),\left(u_{21}, v_{21}\right)$, and $\left(u_{22}, v_{22}\right)$; the edges in $M_{2,2}^{e}$ are $\left(u_{1}, v_{1}\right),\left(u_{2}, v_{5}\right),\left(u_{3}, v_{6}\right),\left(u_{6}, v_{2}\right),\left(u_{7}, v_{7}\right),\left(u_{8}, v_{8}\right),\left(u_{10}, v_{13}\right),\left(u_{11}, v_{14}\right)$, $\left(u_{14}, v_{3}\right),\left(u_{15}, v_{17}\right),\left(u_{16}, v_{18}\right),\left(u_{19}, v_{4}\right),\left(u_{22}, v_{21}\right)$, and $\left(u_{23}, v_{22}\right)$; the edges in $M_{2,2}^{o}$ are $\left(u_{6}, v_{9}\right)$, $\left(u_{7}, v_{10}\right),\left(u_{14}, v_{15}\right),\left(u_{15}, v_{16}\right),\left(u_{19}, v_{19}\right),\left(u_{20}, v_{20}\right),\left(u_{23}, v_{23}\right)$, and $\left(u_{24}, v_{24}\right)$.

Note that for each edge $\left(u_{i}, v_{j}\right) \in M_{2,1}^{o} \cup M_{2,2}^{o}$, we have $v_{j} \notin \mathcal{J}_{1}$. Indeed, $U_{2,1}^{o} \subseteq U_{2,1}$ and $\left.U_{2,2}^{o} \subseteq U_{2,2} \cdot\right]$

5. For the set $\bar{U}_{2,1}^{o}$ of vertices of $U$ that are not matched by $M_{2,1}^{o}$, compute a maximum-weight matching $N_{2,1}^{o}$ between the vertices in $\bar{U}_{2,1}^{o}$ and the vertices in $\mathcal{J}_{1}$.

[Comment: $M_{2,1}^{o} \cup N_{2,1}^{o}$ is a constrained matching in $G$ (cf. Lemma 2.7). In our example (cf. Figures 1 and 7 ), the vertices in $\bar{U}_{2,1}^{o}$ are $u_{1}, \ldots, u_{4}, u_{7}, \ldots, u_{12}, u_{15}, \ldots, u_{17}, u_{20}, u_{23}$, and $u_{24} ; N_{2,1}^{o}=\left\{\left(u_{1}, v_{1}\right),\left(u_{9}, v_{2}\right)\right\}$.]

6. For the set $\bar{U}_{2,2}^{o}$ of vertices of $U$ that are not matched by $M_{2,2}^{o}$, compute a maximum-weight matching $N_{2,2}^{o}$ between the vertices in $\bar{U}_{2,2}^{o}$ and the vertices in $\mathcal{J}_{1}$.

[Comment: $M_{2,2}^{o} \cup N_{2,2}^{o}$ is a constrained matching in $G$ (cf. Lemma 2.7). In our example (cf. Figures 1 and 7 ), the vertices in $\bar{U}_{2,2}^{o}$ are $u_{1}, \ldots, u_{5}, u_{8}, \ldots, u_{13}, u_{16}, \ldots, u_{18}, u_{21}$, and $u_{22}$; $N_{2,2}^{o}=\left\{\left(u_{2}, v_{1}\right),\left(u_{9}, v_{2}\right)\right\}$.]

7. Let $Z_{4}$ be the maximum-weight matching among $M_{2,1}^{e}, M_{2,1}^{o} \cup N_{2,1}^{o}, M_{2,2}^{e}, M_{2,2}^{o} \cup N_{2,2}^{o}$. Output $Z_{4}$.

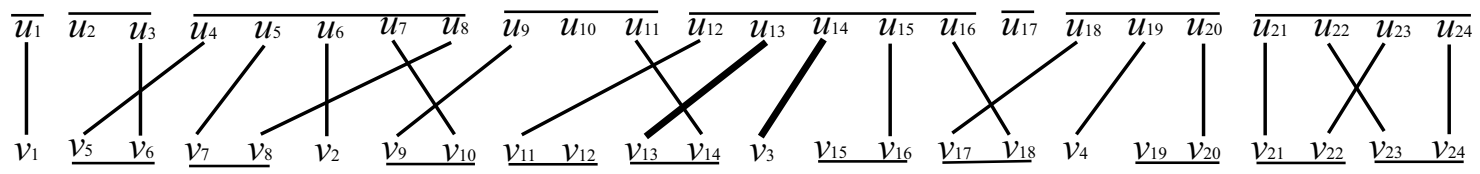

Figure 7: The segments of $U$ obtained from $M_{\text {un }}^{*}$ in Figure 4. Each segment is shown by drawing a common line above the vertices of that segment.

Lemma 2.7 $M_{2,1}^{e}, M_{2,1}^{o} \cup N_{2,1}^{o}, M_{2,2}^{e}$ and $M_{2,2}^{o} \cup N_{2,2}^{o}$ are constrained matchings in $G$.

Proof. Note that $U_{2,1}^{e} \subseteq U-U_{2,2}$. Thus, to prove that $M_{2,1}^{e}$ is a constrained matching in $G$, it suffices to prove that for every $u_{i} \in U_{2,1}^{e} \cap U_{2,1}, u_{i+1} \notin U_{2,1}^{e}$. Consider an arbitrary $u_{i} \in U_{2,1}^{e} \cap U_{2,1}$. By the definition of a segment, $u_{i}$ and $u_{i+1}$ belong to the same segment. So, by the definition of $U_{2,1}^{e}, u_{i+1} \notin U_{2,1}^{e}$. This completes the proof that $M_{2,1}^{e}$ is a constrained matching of $G$. Similarly, we can prove that $M_{2,1}^{o}, M_{2,2}^{e}$ and $M_{2,2}^{o}$ are constrained matchings in $G$. 
To see that $M_{2,1}^{o} \cup N_{2,1}^{o}$ and $M_{2,2}^{o} \cup N_{2,2}^{o}$ are constrained matchings in $G$, first note that $U_{2,1}^{o} \subseteq U_{2,1}$ and $U_{2,2}^{o} \subseteq U_{2,2}$. Thus, for every edge $\left(u_{i}, v_{j}\right) \in M_{2,1}^{o} \cup M_{2,2}^{o}$, we have $v_{j} \notin \mathcal{J}_{1}$. In turn, $M_{2,1}^{o} \cup N_{2,1}^{o}$ is a constrained matching in $G$. Similarly, $M_{2,2}^{o} \cup N_{2,2}^{o}$ is a constrained matching in $G$.

Lemma $2.8 w\left(M_{2,1}^{e}\right)+w\left(M_{2,1}^{o}\right)+w\left(M_{2,2}^{e}\right)+w\left(M_{2,2}^{o}\right) \geq 2 w\left(M_{\mathrm{un}}^{*}\right)$.

Proof. Consider an arbitrary edge $\left(u_{i}, v_{j}\right) \in M_{\text {un }}^{*}$. We distinguish three cases as follows.

Case 1: $u_{i} \in U_{1}$ (i.e., $v_{j} \in \mathcal{J}_{1}$ ). Then, $u_{i}$ must be the center of a segment and hence $u_{i}$ is contained in both $U_{2,1}^{e}$ and $U_{2,2}^{e}$ by Step 3; consequently, edge $\left(u_{i}, v_{j}\right)$ is contained in both $M_{2,1}^{e}$ and $M_{2,2}^{e}$ by Step 4.

Case 2: $u_{i} \in U_{2,1}$ (hence, $v_{j} \in \mathcal{J}_{2}$ ). Then, $u_{i}$ and $u_{i+1}$ must belong to the same segment, say $s$. If $\left(u_{i+1}, v_{j+1}\right)$ is also in $M_{\mathrm{un}}^{*}$, then either $u_{i}$ or $u_{i+1}$ is the center of $s$. In either case, $x_{s}=i$ and $y_{s}=i+1$, and hence $\left(u_{i}, v_{j}\right)$ belongs to both $M_{2,1}^{e}$ and $M_{2,2}^{e}$ by Step 4 (and so does $\left(u_{i+1}, v_{j+1}\right)$ ). On the other hand, if $\left(u_{i+1}, v_{j+1}\right)$ is not in $M_{\mathrm{un}}^{*}$, then either $M_{2,1}^{e}$ or $M_{2,1}^{o}$ contains both $\left(u_{i}, v_{j}\right)$ and $\left(u_{i+1}, v_{j+1}\right)$. Since $\left(u_{i}, v_{j}\right)$ and $\left(u_{i+1}, v_{j+1}\right)$ have the same weight, we can think of $\left(u_{i+1}, v_{j+1}\right)$ as a copy of $\left(u_{i}, v_{j}\right)$.

Case 3: $u_{i} \in U_{2,2}$ (hence, $\left.v_{j} \in \mathcal{J}_{2}^{\prime}\right)$. Similar to Case 2.

By the above case-analysis, we see that for each edge $\left(u_{i}, v_{j}\right)$ of $M_{\mathrm{un}}^{*}$, either $\left(u_{i}, v_{j}\right)$ belongs to two of $M_{2,1}^{e}, M_{2,1}^{o}, M_{2,2}^{e}, M_{2,2}^{o}$, or one of $M_{2,1}^{e}, M_{2,1}^{o}, M_{2,2}^{e}, M_{2,2}^{o}$ contains both $\left(u_{i}, v_{j}\right)$ and its copy. This completes the proof of the lemma.

Lemma $2.9\left(U-\bar{U}_{2,1}^{o}\right) \cap\left(U-\bar{U}_{2,2}^{o}\right) \subseteq W$

Proof. First note that $U-\bar{U}_{2,1}^{o}$ (respectively, $U-\bar{U}_{2,2}^{o}$ ) is the set of vertices in $U$ that are matched by $M_{2,1}^{o}$ (respectively, $M_{2,2}^{o}$ ). Thus, $U-\bar{U}_{2,1}^{o} \subseteq\left\{u_{c(s)-\ell(s)}, \ldots, u_{x(s)-1}, u_{x(s)} \mid s\right.$ is a segment $\}$ and $U-\bar{U}_{2,2}^{o} \subseteq\left\{u_{y(s)}, u_{y(s)+1}, \ldots, u_{c(s)+r(s)} \mid s\right.$ is a segment $\}$. In turn, since segments are disjoint and $x(s) \leq y(s)$ for every segment $s$, it follows that for every $u_{i} \in\left(U-\bar{U}_{2,1}^{o}\right) \cap\left(U-\bar{U}_{2,2}^{o}\right)$, we have $i=x(s)=y(s)$ for some segment $s$. Now, by the definitions of $x(s)$ and $y(s)$, the fact $i=x(s)=y(s)$ implies $u_{i} \in U_{1}$. Moreover, since $u_{i} \in\left(U-\bar{U}_{2,1}^{o}\right) \cap\left(U-\bar{U}_{2,2}^{o}\right), u_{i}$ is matched by $M_{2,1}^{o}$ and so $u_{i-1} \in U_{2,1}$. For the same reason, $u_{i}$ is matched by $M_{2,2}^{o}$ and so $u_{i+1} \in U_{2,2}$.

In summary, for every $u_{i} \in\left(U-\bar{U}_{2,1}^{o}\right) \cap\left(U-\bar{U}_{2,2}^{o}\right)$, we have $u_{i} \in U_{1}, u_{i-1} \in U_{2,1}$, and $u_{i+1} \in U_{2,2}$; hence, $u_{i} \in W$. This completes the proof of the lemma.

\subsection{Performance of the algorithm when $\beta$ is small}

For a contradiction, assume the following:

Assumption $2.10 \beta<\left(\frac{1}{6}+\frac{5}{3} \epsilon\right) w\left(M^{*}\right)$ and $\max \left\{w\left(Z_{1}\right), w\left(Z_{4}\right)\right\}<\left(\frac{1}{2}+\epsilon\right) w\left(M^{*}\right)$.

We want to derive a contradiction under this assumption. First, we derive three inequalities from this assumption and the lemmas in Section 2.5.

Lemma $2.11 w\left(M_{2,1}^{o}\right)+w\left(M_{2,2}^{o}\right) \geq(1-2 \epsilon) w\left(M^{*}\right)$.

Proof. Assume, on the contrary, that $w\left(M_{2,1}^{o}\right)+w\left(M_{2,2}^{o}\right)<(1-2 \epsilon) w\left(M^{*}\right)$. By Lemma 2.8 and the fact that $w\left(M_{\mathrm{un}}^{*}\right) \geq w\left(M^{*}\right)$, we have $w\left(M_{2,1}^{e}\right)+w\left(M_{2,2}^{e}\right) \geq(1+2 \epsilon) w\left(M^{*}\right)$. But then $\max \left\{w\left(M_{2,1}^{e}\right), w\left(M_{2,2}^{e}\right)\right\} \geq\left(\frac{1}{2}+\epsilon\right) w\left(M^{*}\right)$, contradicting Assumption 2.10 .

Lemma $2.12 w\left(N_{2,1}^{o}\right)+w\left(N_{2,2}^{o}\right)<4 \epsilon w\left(M^{*}\right)$. 
Proof. Obviously, $w\left(M_{2,1}^{o} \cup N_{2,1}^{o}\right)+w\left(M_{2,2}^{o} \cup N_{2,2}^{o}\right)=w\left(M_{2,1}^{o}\right)+w\left(M_{2,2}^{o}\right)+w\left(N_{2,1}^{o}\right)+w\left(N_{2,2}^{o}\right)$. By Assumption 2.10, $w\left(M_{2,1}^{o} \cup N_{2,1}^{o}\right)+w\left(M_{2,2}^{o} \cup N_{2,2}^{o}\right)<(1+2 \epsilon) w\left(M^{*}\right)$. So, by Lemma 2.11, $w\left(N_{2,1}^{o}\right)+w\left(N_{2,2}^{o}\right)<4 \epsilon w\left(M^{*}\right)$.

Lemma $2.13 \beta>w_{1}\left(M^{*}\right)-4 \epsilon w\left(M^{*}\right)$.

Proof. Let $\gamma_{1}$ be the total weight of all edges $\left(u_{i}, v_{j}\right) \in M^{*}$ such that $v_{j} \in \mathcal{J}_{1}$ and $u_{i} \in \bar{U}_{2,1}^{o}$. Let $\gamma_{2}$ be the total weight of all edges $\left(u_{i}, v_{j}\right) \in M^{*}$ such that $v_{j} \in \mathcal{J}_{1}$ and $u_{i} \in \bar{U}_{2,2}^{o}$. Let $\gamma_{3}$ be the total weight of all edges $\left(u_{i}, v_{j}\right) \in M^{*}$ such that $v_{j} \in \mathcal{J}_{1}$ and $u_{i} \in\left(U-\bar{U}_{2,1}^{o}\right) \cap\left(U-\bar{U}_{2,2}^{o}\right)$. Clearly, $\gamma_{1}+\gamma_{2}+\gamma_{3} \geq w_{1}\left(M^{*}\right)$. By Steps 5 and 6 in Algorithm $4, \gamma_{1} \leq w\left(N_{2,1}^{o}\right)$ and $\gamma_{2} \leq w\left(N_{2,2}^{o}\right)$. So, by Lemma 2.12, $\gamma_{1}+\gamma_{2}<4 \epsilon w\left(M^{*}\right)$. Moreover, by Lemma $2.9, \beta \geq \gamma_{3}$. Thus, $\beta \geq \gamma_{3} \geq$ $w_{1}\left(M^{*}\right)-\gamma_{1}-\gamma_{2}>w_{1}\left(M^{*}\right)-4 \epsilon w\left(M^{*}\right)$.

Now, we are ready to get a contradiction. By Corollary 2.2 and Assumption 2.10, $w_{1}\left(M^{*}\right)>$ $\left(\frac{1}{2}-3 \epsilon\right) w\left(M^{*}\right)$. Thus, by Lemma $2.13, \beta>\left(\frac{1}{2}-7 \epsilon\right) w\left(M^{*}\right)$. On the other hand, by Assumption 2.10, $\beta<\left(\frac{1}{6}+\frac{5}{3} \epsilon\right) w\left(M^{*}\right)$. Hence,

$$
\frac{1}{2}-7 \epsilon<\frac{1}{6}+\frac{5}{3} \epsilon
$$

contradicting our choice that $\epsilon=\frac{1}{26}$.

Therefore, we have

Theorem 2.14 A constrained matching $Z$ in $G$ with $w(Z) \geq \frac{13}{7} w\left(M^{*}\right)$ can be found in $O\left(I\left(n_{1}+\right.\right.$ $\left.\left.n_{2}\right)\left(I+n_{1}+n_{2}\right)\right)$ time.

\section{2-ISP with a special profit function}

In this section, we consider proportional 2-ISP, where all the positive profits of executing a job are proportional to the lengths of the jobs. A $\frac{5}{3}$-approximation algorithm was recently presented in [4] for proportional 2-ISP. Here, we present a $(1.5+\epsilon)$-approximation algorithm for it for any $\epsilon>0$.

Let $U, \mathcal{J}_{1}$, and $\mathcal{J}_{2}$ be as in Section 2. Let $E$ be the set of those $\left(u_{i}, v_{j}\right) \in U \times \mathcal{J}_{1}$ such that the profit of executing job $v_{j}$ in time unit $u_{i}$ is positive. Let $F$ be the set of those $\left(u_{i}, u_{i+1}, v_{j}\right) \in$ $U \times U \times \mathcal{J}_{2}$ such that the profit of executing job $v_{j}$ in time units $u_{i}$ and $u_{i+1}$ is positive.

Consider the hypergraph $H=\left(U \cup \mathcal{J}_{1} \cup \mathcal{J}_{2}, E \cup F\right)$ on vertex set $U \cup \mathcal{J}_{1} \cup \mathcal{J}_{2}$ and on edge set $E \cup F$. Obviously, proportional 2-ISP becomes the problem of finding a matching $E^{\prime} \cup F^{\prime}$ in $H$ with $E^{\prime} \subseteq E$ and $F^{\prime} \subseteq F$ such that $\left|E^{\prime}\right|+2\left|F^{\prime}\right|$ is maximized over all matchings in $H$. Our idea is to reduce this problem to the problem of finding a maximum cardinality matching in a 3-uniform hypergraph (i.e. each hyperedge consists of exactly three vertices). Since the latter problem admits a $(1.5+\epsilon)$-approximation algorithm [6] and our reduction is approximation preserving, it follows that proportional 2-ISP admits a $(1.5+\epsilon)$-approximation algorithm.

We now detail the approximation-preserving reduction. From $H$, we construct a 3-uniform hypergraph $\mathcal{H}$ as follows. Let

- $\hat{U}=\left\{\hat{u}_{i} \mid u_{i} \in U\right\}$ and $\bar{U}=\left\{\bar{u}_{i} \mid u_{i} \in U\right\}$;

- $\hat{\mathcal{J}}_{2}=\left\{\hat{v}_{j} \mid v_{j} \in \mathcal{J}_{2}\right\}$ and $\overline{\mathcal{J}_{2}}=\left\{\bar{v}_{j} \mid v_{j} \in \mathcal{J}_{2}\right\}$;

- $\tilde{E}=\left\{\left\{\hat{u}_{i}, \bar{u}_{i}, v_{j}\right\} \mid\left(u_{i}, v_{j}\right) \in E\right\}$;

- $\hat{F}=\left\{\left\{\hat{u}_{i}, \hat{u}_{i+1}, \hat{v}_{j}\right\} \mid\left(u_{i}, u_{i+1}, v_{j}\right) \in F\right\}$;

- $\bar{F}=\left\{\left\{\bar{u}_{i}, \bar{u}_{i+1}, \bar{v}_{j}\right\} \mid\left(u_{i}, u_{i+1}, v_{j}\right) \in F\right\}$. 
The vertex set of $\mathcal{H}$ is $\hat{U} \cup \bar{U} \cup \mathcal{J}_{1} \cup \hat{\mathcal{J}}_{2} \cup \overline{\mathcal{J}_{2}}$, and the edge set is $\tilde{E} \cup \hat{F} \cup \bar{F}$.

Lemma 3.1 Let $E^{\prime} \cup F^{\prime}$ with $E^{\prime} \subseteq E$ and $F^{\prime} \subseteq F$ be a matching in $H$. Then, there is a matching in $\mathcal{H}$ of cardinality $\left|E^{\prime}\right|+2\left|F^{\prime}\right|$.

Proof. It suffices to check that $\tilde{E}^{\prime} \cup \hat{F}^{\prime} \cup \overline{F^{\prime}}$ is a matching in $\mathcal{H}$, where $\tilde{E}^{\prime}=\left\{\left\{\hat{u}_{i}, \bar{u}_{i}, v_{j}\right\} \mid\left(u_{i}, v_{j}\right) \in\right.$ $\left.E^{\prime}\right\}, \hat{F}^{\prime}=\left\{\left\{\hat{u}_{i}, \hat{u}_{i+1}, \hat{v}_{j}\right\} \mid\left(u_{i}, u_{i+1}, v_{j}\right) \in F^{\prime}\right\}$, and $\overline{F^{\prime}}=\left\{\left\{\bar{u}_{i}, \bar{u}_{i+1}, \bar{v}_{j}\right\} \mid\left(u_{i}, u_{i+1}, v_{j}\right) \in F^{\prime}\right\}$.

Lemma 3.2 Let $\tilde{E}^{\prime} \cup \hat{F}^{\prime} \cup \bar{F}^{\prime}$ be a matching in $\mathcal{H}$ with $\tilde{E}^{\prime} \subseteq \tilde{E}, \hat{F}^{\prime} \subseteq \hat{F}$ and $\bar{F}^{\prime} \subseteq \bar{F}$. Then, we can compute a matching $E^{\prime} \cup F^{\prime}$ in $H$ with $E^{\prime} \subseteq E, F^{\prime} \subseteq F$, and $\left|E^{\prime}\right|+2\left|F^{\prime}\right| \geq\left|\tilde{E}^{\prime}\right|+\left|\hat{F}^{\prime}\right|+\left|\bar{F}^{\prime}\right|$.

Proof. Let $\tilde{E}^{\prime} \cup \hat{F}^{\prime} \cup \bar{F}^{\prime}$ be a matching in $\mathcal{H}$ as in the lemma. Consider $E^{\prime}=\left\{\left(u_{i}, v_{j}\right) \mid\left\{\hat{u}_{i}, \bar{u}_{i}, v_{j}\right\} \in\right.$ $\left.\tilde{E}^{\prime}\right\}$. Clearly, $E^{\prime}$ is a matching in $H$, that is, no vertex in $U \cup \mathcal{J}_{1}$ belongs to more than one pair in $E^{\prime}$. Moreover, if $u_{i} \in U$ is a vertex belonging to some pair in $E^{\prime}$, then neither $\hat{u}_{i}$ belongs to some triple in $\hat{F}^{\prime}$ nor $\bar{u}_{i}$ belongs to some triple in $\bar{F}^{\prime}$.

Now, either $\left|\hat{F}^{\prime}\right| \geq\left|\bar{F}^{\prime}\right|$ or $\left|\hat{F}^{\prime}\right| \leq\left|\bar{F}^{\prime}\right|$. We assume $\left|\hat{F}^{\prime}\right| \geq\left|\bar{F}^{\prime}\right|$; the other case is similar. Consider $F^{\prime}=\left\{\left(u_{i}, u_{i+1}, v_{j}\right) \mid\left\{\hat{u}_{i}, \hat{u}_{i+1}, \hat{v}_{j}\right\} \in \hat{F}^{\prime}\right\}$. Clearly, $F^{\prime}$ is a matching in $H$, that is, no vertex in $U \cup \mathcal{J}_{2}$ belongs to more than one triple in $F^{\prime}$. Note that $E^{\prime} \cup F^{\prime}$ is also a matching in $H$ and

$$
\left|E^{\prime}\right|+2\left|F^{\prime}\right|=\left|\tilde{E}^{\prime}\right|+2 \max \left\{\left|\hat{F}^{\prime}\right|,\left|\bar{F}^{\prime}\right|\right\} \geq\left|\tilde{E}^{\prime}\right|+\left|\hat{F}^{\prime}\right|+\left|\bar{F}^{\prime}\right|
$$

By the above two lemmas, we have

Theorem 3.3 For every $\epsilon>0$, there is a polynomial-time $(1.5+\epsilon)$-approximation algorithm for proportional 2-ISP.

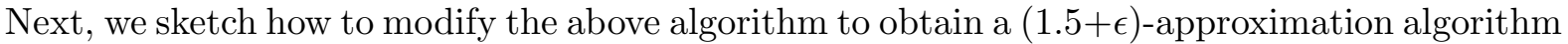
for the unweighted 2-ISP problem. Let $U, \mathcal{J}_{1}$, and $\mathcal{J}_{2}$ be as in Section 2. As in the above, consider the hypergraph $H=\left(U \cup \mathcal{J}_{1} \cup \mathcal{J}_{2}, E \cup F\right)$, where $E$ is the set of those $\left(u_{i}, v_{j}\right) \in U \times \mathcal{J}_{1}$ such that job $v_{j}$ can be executed in time unit $u_{i}$ and $F$ be the set of those $\left(u_{i}, u_{i+1}, v_{j}\right) \in U \times U \times \mathcal{J}_{2}$ such that job $v_{j}$ can be executed in time units $u_{i}$ and $u_{i+1}$. Clearly, unweighted 2-ISP is the problem of finding a maximum cardinality matching $E^{\prime} \cup F^{\prime}$ in $H$ with $E^{\prime} \subseteq E$ and $F^{\prime} \subseteq F$. It is well known [6] that, when the cardinality of all edges of a hypergraph $H$ is bounded by a constant $k$, then the problem of finding a maximum cardinality matching in $H$ admits a $\left(\frac{k}{2}+\epsilon\right)$-approximation algorithm. In this case, we can take $k=3$, which implies a $(1.5+\epsilon)$-approximation algorithm for the unweighted 2-ISP problem.

\section{A new heuristic for protein NMR peak assignment}

As mentioned in Section 1, the $\frac{13}{7}$-approximation algorithm for 2-ISP can be easily incorporated into a heuristic framework for protein NMR peak assignment introduced in [7]. The heuristic first tries to assign "long" segments of three or more spin systems that are under the consecutivity constraint to segments of the host protein sequence, using a simple greedy strategy, and then solves an instance of 2-ISP formed by the remaining unassigned spin systems and amino acids. The first step of the framework is also called greedy filtering and may potentially help improve the accuracy of the heuristic significantly in practice because we are often able to assign long segments of spin systems with high confidence. We have tested the new heuristic based on the $\frac{13}{7}$-approximation algorithm for 2-ISP and compared the results with two of the best approximation and heuristic algorithms in $[3,4,7]$, namely the 2-approximation algorithm for the interval scheduling problem 
$[3,4]$ and the branch-and-bound algorithm (augmented with greedy filtering) $[7]^{6}$. The test data consists of 70 (pseudo) real instances of NMR peak assignment derived from 14 proteins. For each protein, the data of spin systems were from the experimental data in the BioMagResBank database [10], while 5 (density) levels of consecutivity constraints were simulated, as shown in Table 1.

Note that, both the new heuristic algorithm and the 2-approximation algorithm are very fast in general while the branch-and-bound algorithm can be much slower because it may have to explore much of the entire search space. On a standard Linux workstation, it took seconds to hours for each assignment by the branch-and-bound algorithm in the above experiment, while it took a few seconds consistently using either the new heuristic algorithm or the 2-approximation algorithm. Table 1 shows the comparison of the performance of the three algorithms in terms of (i) the weight of the assignment and (ii) the number of correctly assigned spin systems. Although measure (i) is the objective in the interval scheduling problem, measure (ii) is what it counts in NMR peak assignment. Clearly, the new heuristic outperformed the 2-approximation algorithm in both measures by large margins. Furthermore, the new heuristic outperformed the branch-and-bound algorithm in measure (ii), although the branch-and-bound algorithm did slightly better in measure (i). More precisely, the new heuristic was able to assign the same number of or more spin systems correctly than the branch-and-bound algorithm on 53 out of the 70 instances, among which the new heuristic algorithm improved over the branch-and-bound algorithm on 39 instances. ${ }^{7}$ Previously, the branch-and-bound algorithm was known to have the best assignment accuracy (among all heuristics proposed for the interval scheduling problem) [7]. The result demonstrates that this new heuristic based on the $\frac{13}{7}$ approximation algorithm for 2-ISP will be very useful in the automation of NMR peak assignment. In particular, the good assignment accuracy and fast speed allow us to tackle some large-scale problems in experimental NMR peak assignment within realistic computation resources. As an example of application, the consecutivity information derived from experiments may sometimes be ambiguous. The new heuristic algorithm makes it possible for the user to experiment with different interpretations of consecutivity and compare the resulting assignments.

\section{Discussion}

The computational method, presented in this paper, provides a more accurate and more efficient technique for NMR peak assignment, compared to our previous algorithms [3, 4, 7, 9]. We are in the process of incorporating this algorithm into a computational pipeline for fast protein fold recognition and structure determination, using an iterative procedure of NMR peak assignments and protein structure prediction. The basic idea of this pipeline is briefly outlined as follows.

Recent developments in applications of residual dipolar coupling (RDC) data to protein structure determination have indicated that RDC data alone may be adequate for accurate resolution of protein structures [12], bypassing the expensive and time-consuming step of NOE (nuclear Overhauser effect) data collection and assignments. We have recently demonstrated (unpublished results) that if the RDC data/peaks are accurately assigned, we can accurately identify the correct fold of a target protein in the PDB database [11] even when the target protein has lower than $25 \%$ of sequence identity with the corresponding PDB protein of the same structural fold. In addition, we have found that RDC data can be used to accurately rank sequence-fold alignments (alignment accuracy), suggesting the possibility of protein backbone structure prediction by combining RDC data and fold-recognition techniques like protein threading [14].

By including RDC data in our peak assignment algorithm (like [13]), we expect to achieve two things: (a) an improved accuracy of peak assignments with the added information, and (b) an assignment (possibly partial) of the RDC peaks. Using assigned RDC peaks and the aforementioned strategy, we can identify the correct structural folds of a target protein in the PDB database. Then based on the identified structural fold and a computed sequence-fold alignment, we can back-

\footnotetext{
${ }^{6}$ It is worth mentioning that other automated NMR peak assignment programs generally require more NMR experiments, and so they cannot be compared with ours.

${ }^{7}$ It is not completely clear to us why the new heuristic did better on these 39 instances.
} 


\begin{tabular}{|c|c|c|c|c|c|c|c|c|c|c|c|c|c|}
\hline & $W_{1}$ & $R_{1}$ & $W_{2}$ & $R_{2}$ & $W_{3}$ & $R_{3}$ & & $W_{1}$ & $R_{1}$ & $\overline{W_{2}}$ & $R_{2}$ & $\overline{W_{3}}$ & $R_{3}$ \\
\hline bmr4027_5 & 873820 & 40 & 1827498 & 3 & 1934329 & 33 & \multirow{5}{*}{$\begin{array}{l}\text { bmr4144_5 } \\
\text { bmr4144_6 } \\
\text { bmr4144_7 } \\
\text { bmr4144_8 } \\
\text { bmr4144_9 }\end{array}$} & 919419 & 11 & 921816 & 17 & 997603 & 16 \\
\hline bmr4027_6 & 854762 & 64 & 1818131 & 8 & 1921093 & 37 & & 923546 & 21 & 897500 & 11 & 993361 & 11 \\
\hline bmr4027_7 & 1845477 & 89 & 784027 & 44 & 1910897 & 74 & & 954141 & 68 & 842073 & 2 & 954633 & 64 \\
\hline bmr4027_8 & 1900416 & 151 & 1671475 & 19 & 1894532 & 128 & & 953741 & 69 & 804531 & 5 & 954585 & 67 \\
\hline bmr4027_9 & 1896606 & 156 & 1652859 & 60 & 1896606 & 156 & & 952241 & 75 & 837519 & 35 & 952241 & 75 \\
\hline bmr4288_5 & 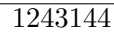 & 36 & 11169907 & 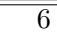 & 1255475 & $\overline{12}$ & \multirow{5}{*}{$\begin{array}{l}\mathrm{bmr} 4302 \_5 \\
\mathrm{bmr} 4302 \_6 \\
\mathrm{bmr} 4302 \_7 \\
\mathrm{bmr} 4302 \_8 \\
\mathrm{bmr} 4302 \_9\end{array}$} & 1275787 & 31 & 1219920 & 11 & 1331391 & 16 \\
\hline $\mathrm{bmr} 4288 \_6$ & 1197106 & 49 & 1179110 & 15 & 1261696 & 26 & & 1282789 & 51 & 174564 & 0 & 1324395 & 43 \\
\hline bmr4288_7 & 1232771 & 65 & 1112288 & 22 & 1251020 & 57 & & 1310324 & 78 & 1181267 & 8 & 1323495 & 62 \\
\hline bmr4288_8 & 1201192 & 68 & 1133554 & 35 & 1238344 & 66 & & 1308217 & 112 & 1152323 & 27 & 1308217 & 103 \\
\hline bmr4288_9 & 1249465 & 105 & 1051817 & 48 & 1249465 & 105 & & 1250300 & 111 & 1293954 & 107 & 1298321 & 110 \\
\hline bmr4309_5 & 1974762 & 35 & 1954955 & 13 & 2117910 & 25 & \multirow{5}{*}{$\begin{array}{l}\mathrm{bmr} 4316 \_5 \\
\mathrm{bmr} 4316 \_6 \\
\mathrm{bmr} 4316 \_7 \\
\mathrm{bmr} 4316 \_8 \\
\mathrm{bmr} 4316 \_9\end{array}$} & 999920 & 43 & 890944 & 2 & 1009329 & 30 \\
\hline bmr4309_6 & 1960424 & 48 & 1924727 & 12 & 2110992 & 57 & & 967526 & 59 & 863207 & 13 & 1022505 & 35 \\
\hline bmr4309_7 & 2046029 & 119 & 1885986 & 24 & 2093595 & 77 & & 925817 & 75 & 882818 & 9 & 1029287 & 79 \\
\hline bmr4309_8 & 1962114 & 121 & 38 & 55 & $20 t$ & 101 & & 1005898 & 75 & 957378 & 62 & 1029287 & 89 \\
\hline bmr4309_9 & 2048987 & 178 & 1796864 & 95 & 2048987 & 178 & & 1029827 & 89 & 984774 & 85 & 1029287 & 89 \\
\hline bmr4s & 2338383 & 19 & $\overline{355926}$ & 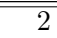 & 249 & 20 & \multirow{5}{*}{$\begin{array}{l}\text { bmr4353_5 } \\
\text { bmr4353_6 } \\
\text { bmr4353_7 } \\
\text { bmr4353_8 } \\
\text { bmr4353_9 }\end{array}$} & 1468772 & $\overline{20}$ & $\begin{array}{l}1417351 \\
\end{array}$ & 8 & $\bar{~} 1532518$ & $\overline{17}$ \\
\hline $\mathrm{bmr} 4$ & 2265090 & 34 & 2312260 & 13 & 248 & 35 & & 1428944 & 23 & 1421633 & 18 & 1524784 & 24 \\
\hline bmr4318_7 & 226 & 73 & 225 & 52 & 24 & 52 & & 1461648 & 56 & 1370235 & 14 & 1516244 & 44 \\
\hline bmr4318_8 & 2217936 & 92 & 2214174 & 63 & 2420829 & 62 & & 1443261 & 78 & 1337329 & 9 & 1472871 & 80 \\
\hline bmr4318_9 & 2339582 & 201 & 2158223 & 122 & 2383453 & 201 & & 1474022 & 124 & 1273988 & 15 & 1483781 & 126 \\
\hline bmr4 & 69 & 10 & 3400 & 5 & 546 & 18 & \multirow{5}{*}{$\begin{array}{l}\text { bmr4393_5 } \\
\text { bmr4393_6 } \\
\text { bmr4393_7 } \\
\text { bmr4393_8 } \\
\text { bmr4393_9 }\end{array}$} & 1816837 & 49 & 1742954 & 3 & 1874095 & 41 \\
\hline bmr4391_6 & 680959 & 7 & 699066 & 8 & 5501 & 10 & & 1843685 & 71 & 1772955 & 42 & 1871616 & 59 \\
\hline bmr4391_7 & 699199 & 17 & 684953 & 37 & 5683 & 26 & & 1847874 & 102 & 1722026 & 22 & 1862221 & 76 \\
\hline bmr4391_8 & 688368 & 38 & 663147 & 30 & 111 & 42 & & 1832576 & 129 & 1709538 & 65 & 1853749 & 130 \\
\hline bmr4391_9 & 710914 & 66 & 687290 & 45 & 710914 & 66 & & 1837340 & 142 & 1527885 & 3 & 1851298 & 152 \\
\hline bmr4579_5 & 913713 & $\overline{18}$ & 894084 & $\overline{22}$ & 967647 & 15 & \multirow{5}{*}{$\begin{array}{l}\text { bmr4670_5 } \\
\text { bmr4670_6 } \\
\text { bmr4670_7 } \\
\text { bmr4670_8 } \\
\text { bmr4670_9 }\end{array}$} & 1365873 & 32 & 1309727 & 11 & 1435721 & 22 \\
\hline bmr4579_6 & 889118 & 35 & 911564 & 8 & 976720 & 32 & & 1326082 & 35 & 1290812 & 13 & 1429449 & 30 \\
\hline bmr4579_7 & 903586 & 48 & 873884 & 17 & 958335 & 44 & & 1353618 & 78 & 1239001 & 6 & 1402335 & 38 \\
\hline bmr4579_8 & 933371 & 72 & 877556 & 26 & 956115 & 63 & & 1391055 & 116 & 1236726 & 19 & 1391055 & 116 \\
\hline bmr4579_9 & 950173 & 86 & 760356 & 0 & 950173 & 86 & & 1391055 & 120 & 1237614 & 60 & 1391055 & 116 \\
\hline bmr4752_5 & 881020 & 21 & 796019 & 8 & 884307 & 21 & \multirow{5}{*}{$\begin{array}{l}\text { bmr4929_5 } \\
\text { bmr4929_6 } \\
\text { bmr4929_7 } \\
\text { bmr4929_8 } \\
\text { bmr4929_9 }\end{array}$} & 1410017 & 17 & 1408112 & 4 & 1496460 & 23 \\
\hline bmr4752_6 & 877313 & 32 & 824289 & 6 & 892520 & 32 & & 1391418 & 36 & 1385673 & 12 & 1496954 & 32 \\
\hline bmr4752_7 & 866896 & 43 & 752633 & 3 & 887292 & 41 & & 1427122 & 69 & 1378166 & 30 & 1490155 & 56 \\
\hline bmr4752_8 & 882755 & 68 & 730276 & 17 & 882755 & 68 & & 1459368 & 82 & 1281548 & 18 & 1481593 & 88 \\
\hline bmr4752_9 & 882755 & 68 & 812950 & 44 & 882755 & 68 & & 1477704 & 114 & 1178499 & 20 & 1477704 & 114 \\
\hline
\end{tabular}

Table 1: The performance of the new heuristic comprising greedy filtering and the $\frac{13}{7}$-approximation algorithm for 2-ISP in comparison with two of the best approximation and heuristic algorithms in $[3,4,7]$ on 70 instances of NMR peak assignment. The protein is represented by the entry name in the BioMagResBank database [10], e.g., bmr4752. The number after the underscore symbol indicates the density level of consecutivity constraints, e.g., _6 means that $60 \%$ of the spin systems are connected to form segments. $W_{1}$ and $R_{1}$ represent the total assignment weight and number of spin systems correctly assigned by the new heuristic, respectively. $W_{2}$ and $R_{2}\left(W_{3}\right.$ and $\left.R_{3}\right)$ are corresponding values for the 2-approximation algorithm for the interval scheduling problem (the branch-and-bound algorithm augmented with greedy filtering, respectively). The numbers in bold indicate that all the spin systems are correctly assigned. The total numbers of spin systems in other proteins are 158 for bmr4027, 215 for bmr4318, 78 for bmr4144, 115 for bmr4302, and 156 for bmr4393.

calculate the theoretical RDC peaks of the predicted backbone structure. Through matching the theoretical and experimental RDC peaks, we can establish an iterative procedure for NMR data assignment and structure prediction. Such a process will iterate until most of the RDC peaks are assigned and a structure is predicted. We expect that such a procedure will prove to be highly effective for fast and accurate protein fold and backbone structure predictions, using NMR data from only a small number of NMR experiments.

\section{References}

[1] A. Bar-Noy, R. Bar-Yehuda, A. Freund, J. Naor, and B. Schieber. A unified approach to approximating resource allocation and scheduling. Journal of the ACM, 48:1069-1090, 2001. 
[2] A. Bar-Noy, S. Guha, J. Naor, and B. Schieber. Approximating the throughput of multiple machines in real-time scheduling. Proceedings of the 31th Annual ACM Symposium on Theory of Computing (STOC'99), pp. 622-631, ACM, 1999.

[3] Z.-Z. Chen, T. Jiang, G. Lin, J. Wen, D. Xu, J. Xu, and Y. Xu. Approximation algorithms for NMR spectral peak assignment. Theoretical Computer Science, 299:211-229, 2003.

[4] Z.-Z. Chen, T. Jiang, G. Lin, J. Wen, D. Xu, and Y. Xu. Improved approximation algorithms for NMR spectral peak assignment. Proceedings of the 2nd Workshop on Algorithms in Bioinformatics (WABI'2002), Lecture Notes in Computer Science, Vol. 2452, pp. 82-96, 2002.

[5] J. Chuzhoy, R. Ostrovsky, and Y. Rabani. Approximation algorithms for the job interval selection problem and related scheduling problems. Proceedings of the 42nd Annual IEEE Symposium on Foundations of Computer Science (FOCS'2001), pp. 348-356, 2001.

[6] C.A.J. Hurkens and A. Schrijver. On the size of systems of sets of every $t$ of which have an SDR, with an application to the worst-case ratio of heuristics for packing problems. SIAM Journal on Discrete Mathematics, 2(1):68-72, 1989.

[7] G. Lin, D. Xu, Z.-Z. Chen, T. Jiang, J. Wen, and Y. Xu. Computational assignments of protein backbone NMR peaks by efficient bounding and filtering. Journal of Bioinformatics and Computational Biology, 31:944-952, 2003.

[8] National Institute of General Medical Sciences. Pilot projects for the protein structure initiative (structural genomics). http://www.nih.gov/grants/guide/rfa-files/RFA-GM-99-009.html, June:RFA GM-99-009, 1999.

[9] Y. Xu, D. Xu, D. Kim, V. Olman, J. Razumovskaya, and T. Jiang. Automated assignment of backbone NMR peaks using constrained bipartite matching. IEEE Computing in Science $\mathcal{G}^{\circ}$ Engineering, 4:50-62, 2002.

[10] University of Wisconsin. BioMagResBank. http://www.bmrb.wisc.edu. University of Wisconsin, Madison, Wisconsin, 2001.

[11] F. C. Bernstein, T. F. Koetzle, G. J. B. Williams, E. F. Meyer, M. D. Brice, J. R. Rodgers, O. Kennard, T. Shimanouchi, and M. Tasumi, The Protein Data Bank: A Computer Based Archival File for Macromolecular Structures, J. Mol. Biol., 112:535 - 542, 1977.

[12] J.C. Hus, D. Marion and M. Blackledge, Determination of protein backbone structure using only residual dipolar couplings, J. Am. Chem. Soc, 123:1541-1542, 2001.

[13] J.C. Hus, J.J. Prompers, and R. Bruschweiler, Assignment strategy for proteins with known structure, Journal of Magnetic Resonnance, 157:119-123, 2002.

[14] Y. Xu and D. Xu, Protein Threading using PROSPECT: design and evaluation, Protein: Structure, Function, Genetics, 40:343 - 354, 2000. 